In summary, consistency between the inertial-frame and comoving-frame equations requires that all $\mathrm{O}(v / c)$ terms be retained in both gas-energy equations, in the radiation energy equation, and in the transformation laws between frames [see also $(\mathbf{P 4})$ ]. In contrast, all $\mathrm{O}(v / c)$ terms can be omitted from the radiation momentum equation without loss of consistency.

Finally, consider the inertial-frame momentum equation (94.13b), which for spherically symmetric flow reduces to

$$
\rho \frac{D v}{D t}=\frac{-G M_{r} \rho}{r^{2}}-\frac{\partial p}{\partial r}-\left[\frac{1}{c^{2}} \frac{\partial F}{\partial t}+\frac{\partial P}{\partial r}+\frac{(3 P-E)}{r}-\frac{v}{c^{2}}\left(\frac{\partial E}{\partial t}+\frac{\partial F}{\partial r}+\frac{2 F}{r}\right)\right] .
$$

On a fluid-flow time scale the term containing $(\partial E / \partial t)$ is $\mathrm{O}\left(v^{2} / c^{2}\right)$ relative to $(\partial P / \partial r)$, and therefore can be dropped. Similarly all terms containing $F$ are at most $\mathrm{O}(v / c)$ relative to the terms in $E$ and $P$. Hence to obtain a final result accurate to $\mathrm{O}(v / c)$ it is sufficient to set $F=F_{0}$, but all terms must be retained in transforming from $(E, P)$ to $\left(E_{0}, P_{0}\right)$. Making these conversions we find

$$
\rho \frac{D v}{D t}=\frac{-G M_{r} \rho}{r^{2}}-\frac{\partial p}{\partial r}-\left[\frac{1}{c^{2}} \frac{\partial F_{0}}{\partial t}+\frac{v}{c^{2}} \frac{\partial F_{0}}{\partial r}+\frac{\partial P_{0}}{\partial r}+\frac{\left(3 P_{0}-E_{0}\right)}{r}+\frac{2}{c^{2}}\left(\frac{\partial v}{\partial r}+\frac{v}{r}\right) F_{0}\right]
$$

which is identical to the comoving-frame equation (96.3). Thus consistency of the momentum equation between frames is assured if, and only if, one accounts for $\mathrm{O}(v / c)$ terms in both frames.

Similarly, in light of (93.10) and (93.11) the inertial-frame momentum equation (94.13a) for a spherically symmetric flow of grey material is

$$
\rho(D v / D t)=-\left(G M_{r} \rho / r^{2}\right)-(\partial p / \partial r)+\left(\kappa_{0} / c\right)[F-(v / c)(E+P)]+\mathrm{O}\left(v^{2} / c^{2}\right)
$$

which, from (91.19), is identical to the comoving-frame equation (96.2) for grey material. Again we see that the $\mathrm{O}(v / c)$ terms are essential for consistency.

\title{
7.3 Solution of the Equations of Radiation Hydrodynamics
}

MATHEMATICAL STRUCTURE OF THE PROBLEM

In $\$ \$ 93$ to 96 we formulated the equations of radiation hydrodynamics in both the Eulerian and Lagrangean frames; we now ask how to solve them. In this connection it is instructive to count the number of variables to be determined and the number of equations available to determine them, as in $\$ 24$. As before we must find seven fluid variables: $\rho, p, T, e$, and three components of $\mathbf{v}$; in addition we must now find ten radiation variables: $E$, the three components of $\mathbf{F}$, and the six nonredundant components of $P$. 
These seventeen variables are related by nine partial differential equations: the equation of continuity, the material energy equation including radiation terms, three components of the material momentum equation, the radiation energy equation, and three components of the radiation momentum equation. In addition we have two material constitutive relations: the pressure and caloric equations of state. (We assume that the material opacity and emissivity are given as functions of, say, $\rho$ and $T$.) We are thus short by six equations, which in effect are closure relations relating $P_{i j}$ to $E$. These relations can be specified a priori in the diffusion regime, but in general they must be determined either iteratively, or from some ad hoc prescription (cf. \$78). In addition we must specify appropriate boundary and initial conditions.

The equations of radiation hydrodynamics in two- and three-dimensional flows are truly formidable; indeed they have never been solved for nontrivial problems except in the limit of radiation diffusion. We shall therefore confine our attention to one-dimensional flows, in which case we need to determine only eight variables $\left(\rho, p, T, e, v_{r}, E, F, P\right)$ from a total of five differential equations and two constitutive relations; here we need to specify only one Eddington factor $f=P / E$ in order to complete the system.

NUMERICAL APPROACH

To handle the coupled system described above we replace the differential equations by suitable discrete approximations and solve these numerically. At this juncture it suffices to describe the procedure verbally, reserving a presentation of difference equations for a specific example in $\$ 98$.

Suppose we wish to solve the Lagrangean equations of radiation hydrodynamics in one-dimensional planar or spherical geometry. We divide the medium into discrete cells, locating velocities at cell interfaces and material properties (density, pressure, etc.) at cell centers, as we did in the absence of radiation (cf. Chapter 5). In the momentum equation we need to know the radiation force (hence the flux) at the same locations as fluid velocities and accelerations (i.e., the interfaces). On the other hand, to apply the first law of thermodynamics to the radiating fluid we need to know the radiation energy density and pressure at the same locations as their material counterparts (i.e., cell centers). Hence we solve the timedependent radiation moment equations on the same mesh as used for the static transfer equation in Chapter 6.

Next consider the time centering of the variables. For simplicity we assume that, as in Chapter 5, we use an explicit form of the momentum equation. As before we time center material properties at $t^{n}$ and velocities at $t^{n-(1 / 2)} \equiv \frac{1}{2}\left(t^{n-1}+t^{n}\right)$. To advance the velocity from $t^{n-(1 / 2)}$ to $t^{n+(1 / 2)}$ we need to know the external forces, the pressure gradient, and the radiation force, all at $t^{n}$; thus radiation forces, whether computed from the flux as in (96.2) or from the gradient of the radiation pressure as in (96.5), should be centered at $t^{n}$. Given velocities $v^{n+(1 / 2)}$ we can advance the interface 
positions from $t^{n}$ to $t^{n+1}$, and by continuity update the material density to $t^{n+1}$. For a nonradiating fluid, we determine $T$, hence $e$ and $p$, at $t^{n+1}$ from an implicit form of the material energy equation. For a radiating fluid, we must solve implicit forms of the material energy equation, including radiation terms, simultaneously with the radiation energy and momentum equations to determine $T, e, p, E, P$, and (if needed) $F$ at $t^{n+1-1}$. Clearly the radiation quantities must be centered at the same time level as the material properties (i.e., at $t^{n}$ and $t^{n+1}$ ).

Integration of the momentum equation including radiation forces differs only trivially from the cases considered in Chapter 5 , hence we focus here primarily on the question of how to formulate and solve the coupled material and radiation energy equations at the advanced time $t^{n+1}$. We discuss first the diffusion limit, then the Lagrangean equations, and finally two Eulerian or mixed-frame approaches. We apply some of these methods in Chapter 8.

\section{Radiation Diffusion Methods}

OVERVIEW

We saw in $\$ 80$ that in static material the radiation field in the equilibrium diffusion regime is determined by local fluid properties and their gradients. We will now show that one can also develop an asymptotic solution of the transfer equation and obtain an explicit analytical expression for the radiation stress-energy tensor in an opaque moving medium. These results afford deep insight into the dynamical effects of radiation in a radiating flow. One can use the analytical form of the stress-energy tensor directly in the energy and momentum equations for the radiating fluid, and thus, in effect, dispense with the transfer equation altogether. We shall treat only nonrelativistic flows; the diffusion approximation in relativistic flows is discussed in (G1). Furthermore we assume pure absorption and ignore scattering; generalizations to include scattering in the Thomson limit can be found in (H2) and (M1), and in the Compton limit in (M2).

The basic assumption made in radiation diffusion theory is that the material is extremely opaque, so that $\left(\lambda_{p} / l\right) \ll 1$. If the material is moving, we must also recognize a second independent small parameter, namely $(v / c) \ll 1$. Our goal is to obtain an expression for the radiation stress-energy tensor by solving the transfer equation through an expansion in terms of these small parameters. As we will see, in the comoving frame (but not the inertial frame) the lowest-order terms that appear in the solution are $O\left(\lambda_{p} / l\right)$, terms of $O(v / c)$ being entirely absent. Thus we can develop a first-order diffusion theory for the comoving-frame radiation field by ignoring fluid motions even in moving material.

The next-higher-order terms will be $\mathrm{O}\left(\lambda_{p} v / l c\right), \mathrm{O}\left(\lambda_{p}^{2} / l^{2}\right)$, and $\mathrm{O}\left(v^{2} / c^{2}\right)$. Of these, the $O\left(\lambda_{p} v / l c\right)$ terms are the most important, being of first order in each of the physically interesting small parameters; we call the expression 
containing these terms the "second-order" solution. Terms of $O\left(\lambda_{p}^{2} / l^{2}\right)$ have never been treated; those of $\mathrm{O}\left(v^{2} / c^{2}\right)$ are contained implicitly in the covariant expression for the stress-energy tensor $R^{\alpha \beta}$ given later. While one expects intuitively the $\mathrm{O}\left(\lambda_{\mathrm{p}} v / l c\right)$ terms to be more important than the other second-order terms, it must be admitted that no rigorous analysis has ever been made to justify this assumption.

One should note that the relative size of the two independent expansion parameters is important because one has static diffusion when $(v / c) \ll\left(\lambda_{p} / l\right)$, so that $t_{d} \ll t_{f}$ and photon diffusion limits the rate of energy flow, and dynamic diffusion when $(v / c) \geq\left(\lambda_{\mathrm{p}} / l\right)$ and advection of energy by the moving fluid sets the effective rate of energy transport (cf. $\$ \$ 93$ and 96).

The assumption of equilibrium is restrictive, and certainly will fail near a boundary surface from which radiation escapes freely. To extend the range of applicability of the theory, one can drop the strong assumption of thermal equilibrium and construct a nonequilibrium diffusion theory that invokes simple relations among the radiation moments, but does not presume that the intensity equals the Planck function at the local material temperature.

In practice it is found that diffusion theory predicts too rapid an energy transport where photon mean free paths become comparable to characteristic structural lengths in the flow. One way to overcome this problem is to introduce flux limiters that restrict the energy transport to physically allowable values. This approach is convenient and has been applied extensively, but lacks the accuracy of a consistent solution of the full transport equation (cf. \$98).

THE ZERO-ORDER AND FIRST-ORDER EQUILIBRIUM DIFFUSION APPROXIMATIONS In a static medium, the radiation field thermalizes to the Planck function as soon as the material is effectively thick. In moving material, the radiation field can achieve local thermal equilibrium only if a photon is destroyed in essentially the same physical environment as it was created, before local conditions are modified significantly by fluid flow. That is, the mean time between absorptions, $t_{\lambda} \approx \lambda_{p} / c$, must be much smaller than a fluid-flow time $t_{f} \approx l / v$, which implies that we must have $\left(\lambda_{p} v / l c\right) \ll 1$, a condition that obviously will be met at great depth in, say, a stellar envelope where both $\left(\lambda_{p} / l\right) \ll 1$ and $(v / c) \ll 1$.

The simplest physical situation is when the material is so homogeneous within an interaction volume that we can neglect all gradients; formally this regime corresponds to the limit $\left(\lambda_{p} / l\right) \rightarrow 0$. Thus, dividing both sides of (95.84) and (95.85) (for grey material) by $\chi_{0}$ and letting $\chi_{0} \rightarrow \infty$ we find that $E_{0}=(4 \pi / c)\left(\eta_{0} / \chi_{0}\right)=(4 \pi / c) B(T)=a_{R} T^{4}$, where $T$ is the material temperature, and that $F_{0} \rightarrow 0$. Moreover, in the absence of gradients the radiation field is isotropic so $P_{0}=\frac{1}{3} E_{0}=\frac{1}{3} a_{R} T^{4}$. Similar arguments applied to $(95.82)$ and $(95.83)$ show that $E_{0}\left(\nu_{0}\right)=3 P_{0}\left(\nu_{0}\right)=(4 \pi / c) B\left(\nu_{0}, T\right)$, and $F_{0}\left(\nu_{0}\right) \rightarrow 0$. Hence a consistent zero-order, comoving-frame radiation 
stress-energy tensor in the equilibrium diffusion limit is

$$
\mathbf{R}_{0}=\left(\begin{array}{cc}
a_{R} T^{4} & \mathbf{0} \\
\mathbf{0} & \frac{1}{3} a_{\mathrm{R}} T^{4} \mathrm{l}
\end{array}\right)
$$

Here 1 denotes the $3 \times 3$ unit matrix. From (97.1) we see that for radiation, just as for an ordinary material gas, transport effects vanish when $\left(\lambda_{p} / l\right) \rightarrow 0$.

To obtain a first-order expression for $R_{0}$ we now assume that terms of $\mathrm{O}\left(\lambda_{\mathrm{p}} / l\right)$ are nonvanishing, but drop all terms of higher order. Consider first the monochromatic radiation momentum equation (95.83) in the isotropic limit, and ignore acceleration terms. Dimensional analysis suggests that on a fluid-flow time scale, all terms containing $F_{0}$ and $Q_{0}$ on the left-hand side are $O\left(\lambda_{p} v / l c\right)$ relative to the term on the right-hand side, and thus can be dropped in a first-order solution. We then obtain

$$
\mathbf{F}_{0}\left(\nu_{0}\right)=-\left[c / \chi_{0}\left(\nu_{0}\right)\right] \nabla \cdot P_{0}\left(\nu_{0}\right)=-\left[4 \pi / 3 \chi_{0}\left(\nu_{0}\right)\right] \nabla B\left(\nu_{0}, T\right),
$$

hence

$$
\mathbf{F}_{0}=-\left(c / 3 \chi_{R}^{0}\right) \nabla\left(a_{R} T^{4}\right)=-\frac{4}{3} a_{R} c \lambda_{R} T^{3} \nabla T \equiv-K_{R} \nabla T,
$$

where $\chi_{R}^{0}$ is the Rosseland mean opacity evaluated in the comoving frame, and $\lambda_{R}=1 / \chi_{R}^{0}$ is the Rosseland mean free path for photons. From (97.3) it is clear that $c^{-1} F_{0}$ is $O\left(\lambda_{0} / l\right)$ relative to $E_{0}$ or $P_{0}$.

It is noteworthy that (97.3), which applies in a moving fluid, is identical in mathematical form to $(80.8)$ for a static medium. Physically this result states that because $\left(\lambda_{p} / l\right) \ll 1$, each fluid element is essentially "unaware" that it is moving, because the radius of the horizon from which it "sees" photons is minuscule compared to the scale of the flow; hence the flux measured in the fluid frame saturates to its static value as determined by the local temperature gradient within the fluid element.

Next consider the radiation energy equation (95.84). Given (97.3), dimensional analysis suggests that all terms on the left-hand side are either $\mathrm{O}\left(\lambda_{\mathrm{p}}^{2} / l^{2}\right)$ or $\mathrm{O}\left(\lambda_{\mathrm{p}} v / l c\right)$ relative to $E_{0}$; hence the departure of $E_{0}$ from $a_{R} T^{4}$ is at most second-order. Therefore a consistent first-order expression for the comoving-frame radiation stress-energy tensor in the equilibrium diffusion regime is

$$
\mathbf{R}_{0}=\left(\begin{array}{cc}
a_{\mathrm{R}} T^{4} & -\frac{1}{3 \chi_{R}} \nabla\left(a_{\mathrm{R}} T^{4}\right) \\
-\frac{1}{3 \chi_{\mathrm{R}}} \nabla\left(a_{\mathrm{R}} T^{4}\right) & \frac{1}{3} a_{\mathrm{R}} T^{4} I
\end{array}\right)=\left(\begin{array}{cc}
a_{R} T^{4} & -\left(K_{R} / c\right) \nabla T \\
-\left(K_{R} / c\right) \nabla T & \frac{1}{3} a_{R} T^{4} \mathbf{I}
\end{array}\right)
$$

We emphasize that (97.4), which contains the standard expressions used in stellar interiors work, applies in the comoving frame only. In particular, (97.3) should not be used as an expression for the inertial-frame flux $F$, which differs from $F_{0}$ by terms that are often much larger than $F_{0}$ itself [cf. (91.17) and (93.13)]. 
Equation (97.4) reveals the interesting fact that to first order the radiation stress-energy tensor contains a "conductive" energy flux, but no viscous stress, in contrast to ordinary gas dynamics where these terms are of the same order. We will see later that radiative viscosity is $O\left(\lambda_{p} v / l c\right)$ relative to $P_{0}$.

By using (97.2) in (96.2), or by writing $P_{0}=\frac{1}{3} E_{0}=\frac{1}{3} a_{R} T^{4}$ and discarding $\mathrm{O}\left(\lambda_{p} v / l c\right)$ terms in (96.3), we see that the comoving-frame momentum equation in the first-order equilibrium diffusion approximation is

$$
\rho(D \mathbf{v} / D t)=\mathbf{f}-\nabla\left(p+\frac{1}{3} a_{R} T^{4}\right) ;
$$

the radiating fluid thus behaves dynamically like an ideal gas whose total pressure is the sum of the gas and radiation pressure (both isotropic). Equation (97.5) is the standard momentum equation used in dynamical stellar structure calculations [see, e.g., (C4, eq. 2), (C5, eq. 27.15), (F1, eq. 2), (K7, eq. 2), (L1, eq. 2.55), or (S3, eq. 3.24)].

The most useful form of the energy equation in the equilibrium diffusion regime is (96.9), the first law of thermodynamics for the radiating fluid. Using the first-order solution (97.4) in (96.9) we find

$$
\frac{D}{D t}\left(e+\frac{a_{R} T^{4}}{\rho}\right)+\left(p+\frac{1}{3} a_{R} T^{4}\right) \frac{D}{D t}\left(\frac{1}{\rho}\right)=\frac{1}{\rho} \nabla \cdot\left(K_{R} \nabla T\right)+\varepsilon,
$$

which for one-dimensional spherically symmetric flow reduces to

$$
\frac{D}{D t}\left(e+\frac{a_{R} T^{4}}{\rho}\right)+\left(p+\frac{1}{3} a_{R} T^{4}\right) \frac{D}{D t}\left(\frac{1}{\rho}\right)=\frac{\partial}{\partial M_{r}}\left[\frac{\left(4 \pi r^{2}\right)^{2} c \rho}{3 \chi_{R}} \frac{\partial\left(a_{R} T^{4}\right)}{\partial M_{r}}\right]+\varepsilon .
$$

At the risk of tedious repetition we again emphasize that all quantities in these equations are measured in the comoving frame. Equation (97.7) is the standard energy equation used in dynamical stellar-structure calculations [see, e.g., (C4, eq. 4), (C5, eq. 27.16), (F1, eq. 4), (K7, eq. 3), (L2, eq. 52.1), or (S3, eq. 3.19)].

Within the framework of one-dimensional Lagrangean hydrodynamics we may regard $\rho$ and $(D \rho / D t)$ as known in the discrete representation of (97.7) spanning the interval $\left(t^{n}, t^{n+1}\right)$. Therefore, (97.7) basically has the mathematical form of a heat conduction equation with nonlinear coefficients. Accordingly, to avoid stringent timestep limitations, we use an implicit (e.g., backward Euler) time-differencing scheme. Then, (97.7) (including artifical viscosity terms-cf. \$59- and with suitable boundary conditions) provides a tridiagonal system of nonlinear equations for $T_{i+(1 / 2)}^{n-1}$ at cell centers. As in $\$ 88$ we linearize this system around some current estimate $T_{i+(1 / 2)}^{*}$, obtaining a system of the form

$$
\begin{aligned}
-A_{i+(1 / 2)} \delta T_{i-(1 / 2)}+B_{i+(1 / 2)} \delta T_{i+(1 / 2)}- & C_{i+\{1 / 2)} \delta T_{i+(3 / 2)} \\
& =R_{i+(1 / 2), \quad(i=1, \ldots, I),}, ~
\end{aligned}
$$

which is solved by Gaussian elimination. This process is iterated to 
convergence. Having obtained $T^{n+1}$, we know $e, p, E_{0}$, and $P_{0}$ at $t^{n+1}$, and can then integrate the momentum equation $(97.5)$ from $t^{n+(1 / 2)}$ to $t^{n+(3 / 2)}$. In short, in the equilibrium diffusion regime the dynamical equations for a radiating fluid are relatively easy to solve.

Finally, we can obtain the inertial-frame radiation stress-energy tensor in the first-order equilibrium diffusion limit by applying the transformations (91.10)-(91.12) to (97.4). To $O(v / c)$ the main effect is to replace the Lagrangean flux by the Eulerian flux, cf. (93.14). Thus using (97.4) and (91.17) in (94.21) one finds that the Eulerian energy equation, correct to $\mathrm{O}(v / c)$, for a radiating fluid in the equilibrium diffusion regime is

$$
\left(\rho e+a_{R} T^{4}\right)_{, t}+\boldsymbol{\nabla} \cdot\left[\left(\rho e+a_{R} T^{4}\right) \mathbf{v}\right]+\left(p+\frac{1}{3} a_{R} T^{4}\right) \boldsymbol{\nabla} \cdot \mathbf{v}=\boldsymbol{\nabla} \cdot\left(K_{R} \boldsymbol{\nabla} T\right)+\rho \varepsilon .
$$

Because the material properties are functions of $(\rho, T),(97.9)$ suffices to determine $T$ at $t^{n+1}$. A simple rearrangement reduces (97.9) to (97.6); the same result is obtained by starting from (94.22) and making $\mathrm{O}(v / c)$ transformations of inertial-frame radiation quantities into the fluid frame [see also (P4)].

THE "SECOND-ORDER" EQUILIBRIUM DIFFUSION APPROXIMATION

In first-order diffusion theory we dropped time derivatives and velocitydependent terms and obtained a radiation stress-energy tensor comprising an energy density, an isotropic pressure, and an energy flux proportional to the local temperature gradient. We now develop the next level of approximation, retaining terms of $\mathrm{O}\left(\lambda_{p} v / l c\right)$; the radiation stress-energy tensor then contains dissipative terms corresponding to radiative viscosity. The main purpose of the discussion is to exhibit the complete one-to-one correspondence that exists between the dynamical behavior of radiation in the diffusion regime and that of a viscous, heat-conducting, relativistic material fluid as described in $\$ 4.3$.

Radiative viscosity was first discussed by Jeans (J1), (J2) and Milne (M14), (M15), who concluded that it provides an efficient mechanism for angular momentum transport in stellar interiors, and promotes solid-body rotation of stars. Their analyses are not relativistically correct, and some of their formulae are flawed. The correct formulae were first derived in a penetrating paper by L. H. Thomas (T1), and later, in manifestly covariant analyses, by Hazelhurst and Sargent (H1) and by Simon (S4).

The approach followed by Thomas is to use (89.5), (90.6), and (90.8) to write the mixed-frame transfer equation in Cartesian coordinates as

$$
\begin{aligned}
{\left[c^{-1}(\partial / \partial t)+\mathbf{n} \cdot \boldsymbol{\nabla}\right] I(\mathbf{n}, \nu) } & =\left(\nu / \nu_{0}\right)^{2} \eta_{0}\left(\nu_{0}\right)-\left(\nu_{0} / \nu\right) \kappa_{0}\left(\nu_{0}\right) I(\mathbf{n}, \nu) \\
= & {\left[\left(1-v^{2} / c^{2}\right) /(1-\mathbf{n} \cdot \mathbf{v} / c)^{2}\right] \eta_{0}\left(\nu_{0}\right) } \\
& -\left[(1-\mathbf{n} \cdot \mathbf{v} / c) /\left(1-v^{2} / c^{2}\right)^{1 / 2}\right] \kappa_{0}\left(\nu_{0}\right) I(\mathbf{n}, \nu),
\end{aligned}
$$

and then, starting from the LTE solution $I=\eta_{0} / \kappa_{0}$, to solve (97.10) by 
iteration, which yields

$$
\begin{aligned}
I(\mathbf{n}, \nu)= & \frac{\left(1-v^{2} / c^{2}\right)^{3 / 2}}{(1-\mathbf{n} \cdot \mathbf{v} / c)^{3}} \frac{\eta_{0}}{\kappa_{0}} \\
& -\frac{\left(1-v^{2} / c^{2}\right)^{1 / 2}}{(1-\mathbf{n} \cdot \mathbf{v} / c)} \frac{1}{\kappa_{0}}\left(\frac{1}{c} \frac{\partial}{\partial t}+\mathbf{n} \cdot \boldsymbol{\nabla}\right) \frac{\left(1-v^{2} / c^{2}\right)^{3 / 2}}{(1-\mathbf{n} \cdot \mathbf{v} / c)^{3}} \frac{\eta_{0}}{\kappa_{0}}+\ldots
\end{aligned}
$$

One can then evaluate R by direct integration of $I(\mathbf{n}, \nu)$ over $\omega$ and $\nu$. The calculation is straightforward, but lengthy and cumbersome, and the rearrangement of terms in the final expression for $R$ (T1, eq. 7 and the unnumbered equation on p. 248) into a covariant form (T1, eqs. 7.1 and 8) is rather tricky.

The most physically appealing approach is Simon's, who used the Eckart decomposition theorem to express $\mathrm{R}$ in terms of quantities that are easily evaluated in the comoving frame; we follow Simon's analysis here. Applying (44.14) to the tensor $W^{\alpha \beta} \equiv c^{2} R^{\alpha \beta}$ we can write

$$
R^{\alpha \beta}=\mathscr{P}^{\alpha \beta}+c^{-2}\left(\mathscr{F}^{\alpha} V^{\beta}+V^{\alpha \mathscr{F}^{\beta}}+\mathscr{E} V^{\alpha} V^{\beta}\right)
$$

where

$$
\begin{aligned}
\mathscr{E} & =c^{-2} V_{\alpha} V_{\beta} R^{\alpha \beta}, \\
\mathscr{F}^{\alpha} & =-S_{\beta}^{\alpha} R^{\beta \gamma} V_{\gamma},
\end{aligned}
$$

and

$$
\mathscr{P}^{\alpha \beta}=S_{\gamma}^{\alpha} S_{\delta}^{\beta} R^{\gamma \delta} .
$$

Here $S_{\beta}^{\alpha}$ is the projection tensor defined by (44.2); we use a different letter for it here to avoid confusion with the radiation pressure tensor $P$.

Because $V_{\alpha}$ is orthogonal to $S_{\beta}^{\alpha}$, it is orthogonal to both $\mathscr{F}^{\alpha}$ and $\mathscr{P}^{\alpha \beta}$, which implies that

$$
\mathscr{F}^{0}=v_{i} \mathscr{F}^{\mathrm{i}} / c \text {, }
$$

and that

and

$$
\mathscr{P}^{i 0}=\mathscr{P}^{i j} v_{i} / c
$$

$$
\mathscr{P}^{00}=\mathscr{P}^{i j} v_{i} v_{j} / c^{2} .
$$

In the comoving frame where $V_{0}^{\alpha}=(c, 0,0,0)$, we thus have

$$
\mathscr{P}_{0}^{\alpha 0}=0 \text {, }
$$

and

$$
\mathscr{F}_{0}^{0}=0
$$

and hence from (97.12)

and

$$
\begin{aligned}
& \mathscr{P}_{0}^{i j}=P_{0}^{i j}=R_{0}^{i j}, \\
& \mathscr{F}_{0}^{i}=F_{0}^{i}=R_{0}^{0 i},
\end{aligned}
$$

$$
\mathscr{E}_{0}=E_{0}=R_{0}^{00} .
$$

These results are completely general. 
Suppose now that in the comoving frame LTE obtains, so that $\eta_{0}\left(\nu_{0}\right)=$ $\kappa_{0}\left(\nu_{0}\right) B\left(\nu_{0}, T\right)$ where $T$ is the material temperature measured in the comoving frame. Then the comoving-frame emissivity can be written in terms of invariants as

$$
e_{0}=a_{0} g_{R}^{0},
$$

where $g_{R}^{0}$ is the invariant photon distribution function for blackbody radiation at rest relative to the observer, that is,

$$
g_{R}^{0}=\left(c^{2} / h^{4} \nu_{0}^{3}\right) B\left(\nu_{0}, T\right)=\left(2 / h^{3}\right)\left[\exp \left(h \nu_{0} / k T\right)-1\right]^{-1},
$$

and $a_{0}$ is the invariant opacity

$$
\omega_{0}\left(\nu_{0}\right)=h \nu_{0} \kappa_{0}\left(\nu_{0}\right)=h \nu_{0} K_{0},
$$

where $K_{0}$ is a world scalar.

In order to generalize (97.18) to an arbitrary frame, we notice that

$$
M_{\alpha} V^{\alpha} \equiv-h \nu_{0}
$$

hence the appropriate covariant generalization of $g_{R}^{0}$ is

$$
g_{R}=\left(2 / h^{3}\right)\left[\exp \left(-M_{\alpha} V^{\alpha} / k T\right)-1\right]^{-1}
$$

and the covariant generalization of $\alpha_{0}$ is

$$
a=\left(-M_{\alpha} V^{\alpha}\right) K_{0} \text {. }
$$

Thus for LTE in the fluid frame the photon Boltzmann equation (92.6) in an arbitrary inertial frame is

$$
M^{\alpha} f_{R, \alpha}=\left(-M_{\alpha} V^{\alpha} / c\right) K_{0}\left(g_{R}-f_{R}\right),
$$

where for convenience we have temporarily adopted Cartesian coordinates.

To obtain an approximation for $f_{R}$, we assume that in the diffusion regime $f_{R}$ does not differ much from $g_{R}$, and use (97.24) to develop the expansion

$$
f_{\mathrm{R}}=g_{R}+\left(c / K_{0} M_{\alpha} V^{\alpha}\right) M^{\alpha} g_{R, \alpha}+\ldots
$$

In (97.25), $g_{R}$ depends on $x^{\alpha}$ because both $T$ and $V^{\alpha}$ are functions of $x^{\alpha}$. We could now follow Thomas and use (97.25) in (91.2) to calculate $R^{\alpha \beta}$ directly. It is much simpler, however, to carry out the calculation in the comoving frame, and then reconstruct $R$ in the inertial frame via (97.12) to (97.17).

Because $V_{\alpha} V_{, \beta}^{\alpha} \equiv 0,\left(V_{, \alpha}^{(0)}\right)_{0} \equiv 0$ in the comoving frame. Therefore in this frame

$$
\begin{aligned}
\left(M^{\alpha} g_{R, \alpha}\right)_{0} & =M_{0}^{\alpha}\left[\left(\partial g_{R} / \partial T\right) T_{, \alpha}+\left(\partial g_{R} / \partial V^{\beta}\right) V_{, \alpha}^{\beta}\right]_{0} \\
& =M_{0}^{\alpha}\left[\left(\partial g_{R}^{0} / \partial T\right)\left(\partial T / \partial x_{0}^{\alpha}\right)+\left(\partial g_{R} / \partial V^{i}\right)_{0}\left(\partial V^{i} / \partial x^{\alpha}\right)_{0}\right] .
\end{aligned}
$$

From (97.20)

$$
\left(\partial g_{R}^{0} / \partial T\right)=\left(c^{2} / h^{4} \nu_{0}^{3}\right)\left[\partial B\left(\nu_{0}, T\right) / \partial T\right]
$$


and from (97.22)

$$
\frac{\partial g_{\mathrm{R}}}{\partial V^{\alpha}}=\left(\frac{2}{h^{3}}\right) \frac{\left(M_{\alpha} / k T\right) \exp \left(-M_{\alpha} V^{\alpha} / k T\right)}{\left[\exp \left(-M_{\alpha} V^{\alpha} / k T\right)-1\right]^{2}},
$$

whence

$$
\left(\partial g_{R} / \partial V^{i}\right)_{0}=\left(T n_{i} / c\right)\left(\partial g_{R}^{0} / \partial T\right)
$$

Substituting (97.26) to (97.29) into (97.25), evaluated in the comoving frame, we obtain

$$
f_{R}^{0}=g_{R}^{0}-\left[\frac{c^{2}}{h^{4} \nu_{0}^{3} \kappa_{0}\left(\nu_{0}\right)} \frac{\partial B\left(\nu_{0}, T\right)}{\partial T}\right] \frac{M_{0}^{\alpha}}{\left|M_{0}^{\alpha}\right|}\left(\frac{\partial T}{\partial x_{0}^{\alpha}}+\frac{T n_{i}^{0}}{c} \frac{\partial V^{i}}{\partial x_{0}^{\alpha}}\right) .
$$

We can now evaluate $R_{0}$ by substituting (97.30) into (91.2). Recalling that $d^{3} M=M^{2} d M d \omega=h^{3} c^{-3} \nu^{2} d \nu d \omega$, we see from (97.17c) and (91.3) that

$$
\begin{aligned}
\mathscr{E}_{0} & =\int_{0}^{\infty} d \nu_{0} \oint d \omega_{0}\left(h^{4} \nu_{0}^{3} / c^{3}\right) f_{\mathrm{R}}^{0} \\
& =\frac{4 \pi}{3}\left[B(T)-\frac{1}{\kappa_{R}^{0}} \frac{\partial B(T)}{\partial T}\left(\frac{\partial T}{\partial x_{0}^{0}}+\frac{T}{c}\left\langle n^{\alpha} n_{i}\right\rangle_{0} \frac{\partial V_{0}^{i}}{\partial x_{0}^{\alpha}}\right)\right] .
\end{aligned}
$$

Here

$$
B(T)=(c / 4 \pi) a_{R} T^{4}
$$

and $\kappa_{R}^{0}$ is the comoving-frame Rosseland mean

$$
\frac{1}{\kappa_{R}^{0}}=\int_{0}^{\infty} \frac{1}{\kappa_{0}\left(\nu_{0}\right)} \frac{\partial B\left(\nu_{0}, T\right)}{\partial T} d \nu_{0} / \int_{0}^{\infty} \frac{\partial B\left(\nu_{0}, T\right)}{\partial T} d \nu_{0},
$$

a world scalar. Noting that

$$
\left\langle n^{\alpha} n_{i}\right\rangle_{0} \equiv(4 \pi)^{-1} \oint n_{0}^{\alpha} n_{i}^{0} d \omega_{0}=\frac{1}{3} \delta_{i}^{\alpha},
$$

we can rewrite (97.31) as

$$
\mathscr{E}_{0}=a_{R} T^{4}-\frac{4}{3}\left(\frac{a_{R} T^{4}}{c \kappa_{R}^{0}}\right)\left(\frac{\partial V_{0}^{i}}{\partial x_{0}^{i}}+\frac{3 c}{T} \frac{\partial T}{\partial x_{0}^{0}}\right) .
$$

Similarly, using (97.30) in (97.17b) and (91.4) we find

$$
\mathscr{F}_{0}^{i}=-\frac{4}{3}\left(\frac{a_{\mathrm{R}} T^{4}}{c \kappa_{R}^{0}}\right)\left(\frac{c^{2}}{T} \frac{\partial T}{\partial x_{0}^{i}}+c \frac{\partial V_{0}^{i}}{\partial x_{0}^{0}}\right) .
$$

Finally, from (97.17a) and (91.1) we obtain

$$
\mathscr{P P}_{0}^{\mathrm{ij}}=\frac{1}{3} a_{\mathrm{R}} T^{4} \delta^{i j}-\frac{4 \pi}{c \kappa_{R}^{0}} \frac{\partial B(T)}{\partial T}\left(\left\langle n^{i} n^{i}\right\rangle \frac{\partial T}{\partial x_{0}^{0}}+\left\langle n^{i} n^{i} n_{k} n^{l}\right\rangle \frac{T}{c} \frac{\partial V_{0}^{k}}{\partial x_{0}^{l}}\right) .
$$

But

$$
\left\langle n^{i} n^{i} n_{k} n^{l}\right\rangle=\frac{1}{15}\left(\delta^{i j} \delta_{k}^{l}+\delta_{k}^{i} \delta^{i l}+\delta^{i l} \delta_{k}^{i}\right)
$$


hence

$$
\mathscr{P}_{0}^{i i}=\frac{1}{3} a_{R} T^{4} \delta^{i j}-\frac{4}{15}\left(\frac{a_{R} T^{4}}{c \kappa_{R}^{0}}\right)\left(\frac{\partial V_{0}^{i}}{\partial x_{0}^{k}} \delta^{k i}+\frac{\partial V_{0}^{i}}{\partial x_{0}^{k}} \delta^{k i}+\frac{\partial V_{0}^{k}}{\partial x_{0}^{k}} \delta^{i j}+\frac{5 c}{T} \frac{\partial T}{\partial x_{0}^{0}} \delta^{i j}\right) .
$$

Equations (97.35), (97.36), and (97.39) apply in the comoving frame. To generalize these expressions to an arbitrary frame, in particular the lab frame, we merely cast them into covariant forms that reduce to the correct results in the comoving frame. Let

$$
\mu_{\mathrm{R}} \equiv \frac{4}{15}\left(a_{R} T^{4} / c \kappa_{R}^{0}\right)=\left(T / 5 c^{2}\right) K_{R}
$$

be the coefficient of radiative viscosity, a world scalar; $K_{R}$ is the radiative conductivity as defined in (97.3). From (97.35) we see by inspection that a covariant expression for $\mathscr{E}$ is

$$
\mathscr{E}=a_{R} T^{4}-5 \mu_{R}\left[V_{; \alpha}^{\alpha}+3 V^{\alpha}(\ln T)_{, \alpha}\right] .
$$

To write a covariant expression for $\mathscr{F}^{\alpha}$ we note from (97.16b) and (97.36) that the comoving-frame flux has nonvanishing space components but a vanishing time component. We can assure this behavior by writing $\mathscr{F}^{\alpha}$ in terms of the projection tensor as

$$
\mathscr{F}^{\alpha}=-K_{R}\left(T_{\beta^{\beta}}+c^{-2} T A_{\beta}\right)\left(g^{\alpha \beta}+c^{-2} V^{\alpha} V^{\beta}\right) .
$$

This expression is exactly analogous to Eckart's covariant material heatconduction vector (46.22); it differs from Simon's (S4) equation (82), which is not. By similar reasoning, we replace the Kronecker deltas in (97.39) with projection tensors, and noting that

$$
\left(V^{\alpha} V^{\beta} V^{\gamma}\right)_{; \gamma}=V^{\alpha} V^{\beta} V_{; \gamma}^{\gamma}+V^{\alpha} V^{\gamma} V_{; \gamma}^{\beta}+V^{\beta} V^{\gamma} V_{; \gamma}^{\alpha}
$$

we find that (97.39) can be written covariantly as

$$
\begin{aligned}
\mathscr{P} \alpha \beta= & \frac{1}{3} a_{R} T^{4}\left(g^{\alpha \beta}+c^{-2} V^{\alpha} V^{\beta}\right)-\mu_{R}\left[g^{\alpha \beta} V_{i \gamma}^{\gamma}+g^{\beta \gamma} V_{i \gamma}^{\alpha}+g^{\alpha \gamma} V_{; \gamma}^{\beta}\right. \\
& \left.+c^{-2}\left(V^{\alpha} V^{\beta} V^{\gamma}\right)_{i \gamma}+5 V^{\gamma}(\ln T)_{, \gamma}\left(g^{\alpha \beta}+c^{-2} V^{\alpha} V^{\beta}\right)\right] .
\end{aligned}
$$

Finally, substituting (97.41), (97.42), and (97.44) into (97.12) we find that the covariant expression for the diffusion-limit radiation stress-energy tensor in an arbitrary frame is

$$
\begin{aligned}
R^{\alpha \beta}= & \frac{1}{3} a_{R} T^{4}\left(g^{\alpha \beta}+4 c^{-2} V^{\alpha} V^{\beta}\right)-\mu_{R}\left[g^{\alpha \beta} V_{; \gamma}^{\gamma}+g^{\beta \gamma} V_{; \gamma}^{\alpha}+g^{\alpha \gamma} V_{; \gamma}^{\beta}\right. \\
& +6 c^{-2}\left(V^{\alpha} V^{\beta} V^{\gamma}\right)_{; \gamma} \\
& \left.+5(\ln T)_{; \gamma}\left(g^{\alpha \beta} V^{\gamma}+g^{\beta \gamma} V^{\alpha}+g^{\alpha \gamma} V^{\beta}+6 c^{-2} V^{\alpha} V^{\beta} V^{\gamma}\right)\right] .
\end{aligned}
$$

Aside from minor differences in notation, this result is identical to Thomas's (T1). Equation (97.45) is the direct analogue, for radiation, of (45.3) for matter.

Equations (97.41), (97.42), (97.44), and (97.45) are relativistically correct, and apply in all frames. To gain physical insight it is most instructive 
to work in the comoving frame, developing a solution that is internally consistent to $\mathrm{O}\left(\lambda_{p} v / l c\right)$. Thus, reducing (97.41) to (97.44) to the comoving frame, we find [cf. (97.34) to (97.39)]

and

$$
\begin{aligned}
E_{0} & =a_{R} T^{4}-5 \mu_{R}[3(D \ln T / D t)+\nabla \cdot \mathbf{v}], \\
\left(F_{0}\right)_{i} & =-K_{R}\left(T_{, i}+c^{-2} T a_{i}\right),
\end{aligned}
$$

$$
P_{0}^{i j}=\left[\frac{1}{3} a_{R} T^{4}-5 \mu_{R}(D \ln T / D t)\right] \delta^{i j}-\mu_{R}\left(v_{. k}^{i} \delta^{k j}+v_{, k}^{j} \delta^{i k}+v_{, k}^{k} \delta^{i j}\right) .
$$

We can cast (97.48) into the same form as the stress tensor for a Newtonian fluid [cf. (25.3)], that is

$$
P_{0}^{i i}=\mathscr{P} \delta^{i i}-2 \mu_{R} D^{i j}-\zeta_{R} v_{, k}^{k} \delta^{i j}
$$

where

$$
\mathscr{P} \equiv \frac{1}{3} a_{R} T^{4}-5 \mu_{R}(D \ln T / D t)
$$

is the isotropic component of the radiation pressure, $D^{i i}$ is the traceless rate-of-strain tensor (32.34), and

$$
\zeta_{R} \equiv \frac{5}{3} \mu_{R}
$$

It is obvious from (97.49) why $\mu_{R}$ is called the viscosity coefficient for radiation.

The corresponding results in curvilinear coordinates are obtained by replacing $\delta_{i j}$ with $g_{i j}$ and ordinary derivatives with covariant derivatives.

A dimensional analysis of (97.46) and (97.50) suggests that the departures of $E_{0}$ from $a_{R} T^{4}$ and of $\mathscr{P}$ from $\frac{1}{3} a_{R} T^{4}$ are both $\mathrm{O}\left(\lambda_{\mathrm{p}} v / l c\right)$ relative to the dominant terms; hence when $v / c \gg \lambda_{p} / l$ these departures can be larger than in a static medium [where they are $\mathrm{O}\left(\lambda_{p}^{2} / l^{2}\right)$, cf. $\$ 80$ ]. The radiative viscous terms in (97.49) are also $O\left(\lambda_{p} v / l c\right)$ relative to the leading term. Furthermore, from (97.46) we can now verify the result cited in $\$ \$ 93$ and 95 that the net absorption-emission term $\kappa(4 \pi B-c E)$ in the radiation energy equation is $O(v / l) E$ for dynamic diffusion [compared to $O\left(c \lambda_{p} / l^{2}\right) E$ for static diffusion]. Analysis of (97.47) suggests that the acceleration term is only $\mathrm{O}\left(v^{2} / c^{2}\right)$ relative to the leading term of the flux. Normally we would drop a term of this order, but we retain it here for reasons that will emerge below.

Radiative viscosity arises because photons deposit their momentum in the fluid element in which they are absorbed; it is thus a direct analogue of molecular viscosity. In both cases, momentum exchange via particle motions within the fluid produces a frictional force, with faster-moving elements tending to drag along slower elements, and vice versa. Indeed, it is easy to derive the coefficient of radiative viscosity from simple meanfree-path arguments. Consider photons emitted from the origin $O$, traveling along the positive $x$ axis until they are absorbed at $P$, one mean free path $\lambda_{p}$ from $O$. Let the material have a velocity shear $(d v / d x)$ parallel to 
the $y$ axis. Then, owing to aberration, photons of frequency $\nu$ emitted from $O$ will have a $y$ component of momentum $p_{y}=-(h \nu / c) \sin \theta$, where $\sin \theta=$ $v_{p} / c=\lambda_{p}(d v / d x) / c$, when they are absorbed at $P$. Hence the radiative viscous force on a fluid element at $P$ with cross section $\delta A$ perpendicular to the $x$ axis is $f_{\mathrm{y}}(\nu)=-\left(c n_{\nu} \delta A\right) \times(h \nu / c) \times\left(\lambda_{p} / c\right)(d v / d x)$, where $n_{i}$ is the photon number density. Summing over all energies we find $f_{y}=$ $-\left(a_{R} T^{4} \lambda_{p} / c\right)(d v / d x)$, whence we identify $\mu_{R} \sim\left(a_{R} T^{4} \lambda_{p} / c\right)$, which, aside from a numerical factor, is (97.40).

One can define a radiative Reynolds number $\operatorname{Re}_{R}$ by substituting $\mu_{R}$ into (28.1). Dimensional analysis suggests that $\operatorname{Re}_{R} \sim\left(t_{f} / t_{\lambda}\right) \times$ (material kinetic energy density/radiation energy density). Thus the radiative Reynolds number can be small, hence radiative viscous effects important, when the radiant energy density is large compared to the material energy density (i.e., at high temperatures) and/or when the photon mean free path is long.

From (97.49) and (97.51) it also appears that radiation has a substantial bulk viscosity $\zeta_{R}$. As emphasized by Weinberg (W1) it is necessary to make a deeper inquiry about this quantity. In particular he notes the proof by Tisza (T2) that bulk viscosity is absent in any gas for which the trace of the total stress-energy tensor is expressible as a function of only $\rho_{00} c^{2}$, the total energy density, and/or $n$, the particle number density. This is the case for a gas of structureless point particles in both the nonrelativistic and extreme relativistic limits [cf. (43.50) and (43.53)], hence for a gas of photons. Therefore one would expect photons to have zero bulk viscosity when interacting with relativistic material particles, in apparent contradiction to (97.51). Moreover, according to (97.46), the comoving-frame energy density contains dissipative terms, in contradiction to the general result from Eckart's theory (cf. $\$ \$ 44$ and 45 ) that

$$
\left(M_{\mathrm{viscous}}^{\alpha \beta}+M_{\text {conduction }}^{\alpha \beta}\right) V_{\alpha} V_{\beta} \equiv 0 \text {. }
$$

Weinberg demonstrates that these apparent contradictions can be resolved by noticing that while Thomas and Eckart define particle number density in the same way, they use different definitions for the temperature. Thomas uses the material temperature $T$ measured by a comoving observer, whereas Eckart defines a temperature $T_{*}$ by the requirement that the comoving-frame energy density $c^{-2} V_{\alpha} V_{\beta} M^{\alpha \beta}$ equal the total energy density $\hat{e}=c^{2} \rho_{00}\left(n, T_{*}\right)$ in thermal equilibrium at temperature $T_{*}$. Weinberg shows that

$$
T-T^{*}=15 \mu_{\mathrm{R}}\left(\frac{\partial \hat{e}}{\partial T}\right)_{n}^{-1}\left[\frac{1}{3}-\frac{(\partial p / \partial T)_{n}}{(\partial \hat{e} / \partial T)_{n}}\right] \boldsymbol{\nabla} \cdot \mathbf{v},
$$

that when this difference is taken into account, Thomas's results are consistent with Eckart's general theory, and that in (97.49) one now has

and

$$
\mu_{R}=\frac{4}{15}\left(a_{R} T_{*}^{4} / c \kappa_{R}^{0}\right)
$$

$$
\zeta_{R}=15 \mu_{R}\left[(\partial p / \partial \hat{e})_{n}-\frac{1}{3}\right]^{2} .
$$


From (43.50) and (43.53) we see that (97.55) yields zero radiative bulk viscosity when the radiation interacts with extremely relativistic material, and reduces to Thomas's expression when the material is nonrelativistic.

The physical essence of the preceding analysis is that in the diffusion regime a radiating fluid behaves, in the comoving frame, like a viscous, heat-conducting gas that has a total "internal" energy density (per gram)

$$
e_{\mathrm{tot}}=e+\left(\mathrm{E}_{0} / \rho_{0}\right)
$$

a total hydrostatic pressure

$$
p_{\text {tot }}=p+P,
$$

a total energy flux

$$
\mathbf{q}_{\mathrm{tot}}=\mathbf{q}+\mathbf{F}_{0}=-\left(K+K_{R}\right)\left(\boldsymbol{\nabla} T+c^{-2} T \mathbf{a}\right),
$$

and a total viscous stress tensor

$$
\sigma_{\mathrm{tot}}^{i j}=\left(\mu+\mu_{R}\right)\left(v_{, k}^{i} \delta^{i k}+v_{, k}^{i} \delta^{i k}-\frac{2}{3} v_{, k}^{k} \delta^{i j}\right)+\zeta_{R} v_{, k}^{k} \delta^{i j} .
$$

Adopting this view, we should be able to obtain valid momentum and gas-energy equations for the radiating fluid using the analysis of $\$ \$ 46$ and 47 for a nonideal relativistic fluid. For example, reduction of the momentum equation (47.3) to $\mathrm{O}(v / c)$ in the comoving frame in a spherically symmetric flow gives

$$
\begin{aligned}
& {\left[\rho+\frac{1}{c^{2}}\left(\rho e+p+E_{0}+\mathscr{P}\right)\right] a_{r}=f_{r}-\frac{\partial}{\partial r}(p+\mathscr{P})} \\
& \quad+\frac{\partial}{\partial r}\left[\frac{4}{3}\left(\mu+\mu_{R}\right) r \frac{\partial}{\partial r}\left(\frac{v}{r}\right)+\zeta_{R}\left(\frac{\partial v}{\partial r}+\frac{2 v}{r}\right)\right]+4\left(\mu+\mu_{R}\right) \frac{\partial}{\partial r}\left(\frac{v}{r}\right) \\
& \quad-\frac{1}{c^{2}} \frac{D}{D t}\left(F_{0}+q\right)-\frac{2}{c^{2}}\left(\frac{\partial v}{\partial r}+\frac{v}{r}\right)\left(F_{0}+q\right) .
\end{aligned}
$$

Here we ignored material bulk viscosity, and dropped one term, $-\left(c^{-2} \zeta \nabla \cdot \mathbf{v}\right) a_{r}$, which is formally $\mathrm{O}\left(v^{2} / c^{2}\right)$.

We can check (97.60) by comparing it to (96.1) (to which material viscous and conduction terms are added) in which the comoving-frame radiative force term $\mathbf{G}_{0}$ is evaluated using (95.88) or (95.85), with $E_{0}, F_{0}$, and $P_{0}$ obtained from (97.46) to (97.50). Thus computing the $r r$ component of $P_{0}$ from (97.49) we find

$$
P_{0}=\mathscr{P}-\frac{4}{3} \mu_{R}[(\partial v / \partial r)-(v / r)]-\zeta_{R} \nabla \cdot \mathbf{v},
$$

whence, from (97.46) and (97.50), we have

$$
\left(3 P_{0}-E_{0}\right) / r=-4 \mu_{R}[\partial(v / r) / \partial r] .
$$

Using (97.61) and (97.62) in (95.85) we reproduce the radiative terms in (97.60) exactly, which is very satisfying.

Furthermore, we can now give a precise physical interpretation of the 
acceleration terms in (95.88): they account for the equivalent inertia of the radiation enthalpy density, playing the same role as the material enthalpy terms in $\rho_{000}$ in (96.1). Similarly the terms $c^{-2}\left[\mathbf{F}_{0} \cdot(\boldsymbol{\nabla v})+\mathbf{F}_{0}(\boldsymbol{\nabla} \cdot \mathbf{v})\right]$ in (95.88) account for the interaction of the radiation momentum density with the shear-flow field, playing the same role as the last three terms in (47.3), which account for the inertia of the material heat flux. Finally, $c^{-2}\left(D F_{0} / D t\right)$ accounts for the rate of change of the momentum density of the radiation field [cf. (96.4)], and plays essentially the same role as $\rho a_{r}$ for the matter.

Dimensional analysis of (97.60) suggests that $c^{-2}\left(E_{0}+\mathscr{P}\right) a_{\mathrm{r}}$ is $\mathrm{O}\left(v^{2} / c^{2}\right)$ relative to $\partial \mathscr{P} / \partial r$, while all of the radiation viscosity and flux terms on the right-hand side of $(97.60)$ are $O\left(\lambda_{p} v / l c\right)$. The former can thus be dropped (along with the corresponding material terms) because we have already omitted all other terms that are formally $\mathrm{O}\left(v^{2} / c^{2}\right)$, while all of the latter must be retained if the treatment of radiation viscosity effects in the momentum equation is to be consistent.

Similarly, reducing the gas energy equation (46.15) to $O(v / c)$ in the comoving frame with the help of (46.7) and adding a thermonuclear energy-release term we find that

$$
\begin{aligned}
& \rho\left[\frac{D}{D t}\left(e+\frac{E_{0}}{\rho}\right)+(p+\mathscr{P}) \frac{D}{D t}\left(\frac{1}{\rho}\right)\right] \\
& \quad=\rho \varepsilon_{0}+\Phi-\nabla \cdot\left(\mathbf{q}+\mathbf{F}_{0}\right)-\frac{2}{c^{2}} \mathbf{a} \cdot\left(\mathbf{q}+\mathbf{F}_{0}\right)+\frac{\left(K+K_{R}\right)}{c^{2}} \nabla \cdot \mathbf{v} \frac{D T}{D t}
\end{aligned}
$$

where $\Phi$ is the total dissipation function

$$
\Phi \equiv 2\left(\mu+\mu_{R}\right) D_{i j} D^{i j}+\zeta_{R}(\boldsymbol{\nabla} \cdot \mathbf{v})^{2} .
$$

Again, we have ignored the bulk viscosity of the material.

We can check (97.63) for spherically symmetric flow by comparing it to (96.6), to which we add material viscous and conduction terms, and calculate the radiation energy-deposition rate $c G_{0}^{0}$ from (95.87) or $(95.84)$. Thus using (97.61) and (97.62) we recover all the radiative terms in (97.63) except the last term on the right-hand side. The origin of the discrepancy is obscure, but dimensional analysis suggests that the term in question is formally only $\mathrm{O}\left(v^{2} / c^{2}\right)$ relative to $\boldsymbol{\nabla} \cdot \mathbf{F}_{0}$ and could therefore be neglected (but see below). The term may correspond to a high-order term omitted from (95.87), which is formally accurate only to $\mathrm{O}(v / c)$.

From (97.63) and (97.64) we see that radiation generates entropy in a radiating fluid both by energy transport down a temperature gradient and by viscous dissipation, in complete parallelism with the corresponding material processes. Furthermore, we can now interpret the $\mathbf{a} \cdot \mathbf{F}_{0}$ term in the comoving-frame radiation energy equation physically as accounting for the equivalent inertia of the radiant energy flow, playing the same role as the inertia of thermal heat conduction in material (cf. \$46). 
Dimensional analysis of (97.63) suggests that the terms containing $\mu_{R}$ in (97.46) and (97.48), and the acceleration term in (97.47), all give rise to terms in (97.63) that are of the same order as $\Phi$ and the term containing $\mathbf{a} \cdot \mathbf{F}_{0}$. Thus if we wish to study the effects of radiative viscous dissipation we should retain all terms in (97.46) to (97.48) and in (97.63) for consistency. However, at this point we must note the disturbing fact that the dimensional analysis suggest that all of the radiative dissipative terms in (97.63) are formally only $\mathrm{O}\left(v^{2} / c^{2}\right)$ relative to the leading term $\boldsymbol{\nabla} \cdot \mathbf{F}_{0}$ !

This result may indicate that $(97.63)$ is inadequate to describe radiative viscous effects because neither the comoving-frame radiation energy equation, nor (97.46) to (97.48) for $\mathbf{R}_{0}$, are formally accurate to $\mathrm{O}\left(v^{2} / c^{2}\right)$ and, furthermore, $\mathrm{O}\left(v^{2} / c^{2}\right)$ effects have also been omitted from the material terms. Actually the situation is completely analogous to that discussed in $\$ 51$ for the damping of acoustic waves by material viscosity and thermal conduction: viscous terms affect the momentum balance (whereas conduction terms do not) while conduction terms dominate the energy dissipation (whereas viscous terms are negligible because they are of second order in the velocity perturbation).

The dimensional analysis used above may not be accurate in every case, and for some flows the radiative dissipative terms may be larger, see (M7). But in any event, we conclude that radiative viscous dissipation is generally small for nonrelativistic fluids, in harmony with the conclusions of Cox $(\mathbf{C 5}$, $\$ 27.6 \mathrm{~d})$ and Kopal (K5), (K6) that the effects of both radiative and material viscosity on stellar pulsation are negligible compared to "turbulent viscosity" produced by convective motions in stellar envelopes.

Equations (97.46) to (97.51) provide an accurate radiation stress-energy tensor in the diffusion limit, as needed for, say, dynamical stellar evolution calculations. But it would be laborious to implement the full equations in a computation, and it is natural to ask whether any terms can be dropped in practical applications. A variety of simplified equations for treating radiative viscous effects have been proposed. For example, Simon (S4) suggested that the $(D / D t)$ terms can be dropped in both (97.46) and (97.48). This omission is unsatisfactory in general because dimensional analysis suggests that all the terms containing $\mu_{R}$ in these equations are of the same order. Therefore, if we choose to retain the viscous stress in (97.49), we must retain $(D T / D t)$ in $(97.50)$; we then must retain all terms in (97.46) to guarantee that trace $P_{0}=E_{0}$. More revealing, using (97.46) to (97.48) in the comoving-frame radiation energy equation (95.84) for a planar, grey medium we find that we must retain all terms in (97.46) in order to obtain internal consistency to $\mathrm{O}\left(\lambda_{p} v / l c\right)$; in particular, omission of the $(D / D t)$ term in (97.46) is tantamount to dropping $\left(D E_{0} / D t\right)$ in (95.84), which is obviously unacceptable.

Similar criticisms may be leveled at simplified forms of the momentum and energy equations that have been derived by Newtonian reasoning. For example, in the two standard works on stellar pulsation and stability by 
Ledoux (L1) and Ledoux and Walraven (L2), the momentum equation for the radiating fluid is taken to be

$$
\rho\left(D v_{i} / D t\right)=f_{i}+\left(p+p_{R}\right)_{, i}+g_{i j} \sigma_{; k}^{j k}
$$

where $\sigma^{i i}$ is the total viscous stress tensor (97.59) [cf. eqs. (2.14) and (2.55) of $(\mathbf{L 1})$ and eqs. (48.4) and (50.1) of $(\mathbf{L 2})$ ]. The first law of thermodynamics for the radiating fluid is written

$$
\rho\left[\frac{D}{D t}\left(e+\frac{E_{R}}{\rho}\right)+\left(p+p_{R}\right) \frac{D}{D t}\left(\frac{1}{\rho}\right)\right]=\rho \varepsilon-\nabla \cdot\left(\mathbf{q}+\mathbf{F}_{R}\right)+\Phi
$$

where $\Phi$ is given by (97.64) [see the equation following (2.62) in (L1) and eq. (52.1) in (L2)]. In (L2), $E_{R} \equiv a_{R} T^{4}$ [cf. their eqs. (49.37) and (51.1)], while $p_{R}$ includes dissipative terms [cf. their eq. (49.43)]. In (L1), $E_{R} \equiv$ $a_{R} T^{4}$ and $p_{R} \equiv \frac{1}{3} a_{R} T^{4}$ [cf. eqs. (2.4) and (2.14) of that reference]. In both (L1) and (L2) the acceleration term in (97.47) is dropped from $\mathbf{F}_{R}$.

Unfortunately, all of the simplifications just described neglect terms that are of the same order as the viscous terms that are retained. For example, (97.65) omits the rate of change of the radiation momentum density and the dynamical interaction of the radiation momentum with the velocity gradient, even though these terms are formally of the same order as the viscous stress; it is not, therefore, a consistent equation of motion, and (97.60) should be used instead. Similarly, (97.66) omits terms from $E_{R}, F_{R}$, and $p_{R}$, as well as $\mathbf{a} \cdot \mathbf{F}_{R}$, which are all formally of the same order as $\Phi$, and this equation is not consistent at the level at which radiative dissipative effects enter.

The important conclusion that can be drawn from the discussion above is that all dynamical effects of radiation, including radiative viscosity, inertia of the radiant heat flux and enthalpy density, and radiation momentum density are correctly described by the comoving-frame radiation momentum and energy equations (95.87) and (95.88), provided that all terms in those equations are retained. Thus one can account for these dynamical effects by solving the (full) comoving-frame equations directly, without resort to the second-order diffusion approximation, which supplies analytical results valid only in the limit of small photon mean free paths. In practice, it may be difficult to retain sufficient numerical accuracy to calculate the dissipation terms with any significance at great depth, but it is just then that the departures from adiabaticity they produce are small, perhaps $\mathrm{O}\left(v^{2} / c^{2}\right)$. On the other hand, in the transport regime the comoving-frame moment equations provide a direct means of handling radiative momentum input into, and dissipation in, the material in radiation-dominated flows, just when these effects are large. Analytical expressions for viscous terms cannot be written in the transport regime because they depend on the global structure of the velocity field over a photon mean free path (now comparable to a characteristic structural length) instead of on local velocity gradients. The "viscous" effects are now 
just part of the nonlocal momentum and energy transport calculated from the numerical solution of the radiation moment equations.

THE NONEQUILIBRIUM DIFFUSION APPROXIMATION

One of the assumptions made by equilibrium diffusion theory is that the radiation field and the material are in thermal equilibrium, which implies that the radiation has a Planckian distribution at the material temperature. This assumption is unnecessarily restrictive because problems arise in which the material is opaque, but the matter and radiation are not in equilibrium, for example when the material energy balance is driven by hydrodynamical processes faster than it can relax radiatively, or when the radiation field varies too rapidly for the material to follow instantaneously. It is therefore productive to construct a nonequilibrium diffusion theory in which the radiation field can have an arbitrary spectral distribution and energy density. Interesting early discussions of this approach appear in (C1), (C2), and (F3).*

It is most natural to formulate the theory in the Lagrangean frame, for that is the frame in which the radiation field isotropizes and $P_{0}\left(\nu_{0}\right) \rightarrow$ $\frac{1}{3} E_{0}\left(\nu_{0}\right)$ when $\lambda_{p} \rightarrow 0$. Thus, assuming isotropy in (95.83) and dropping all terms of $\mathrm{O}\left(\lambda_{p} v / l c\right)$ and higher, we have the nonequilibrium diffusion flux

$$
\mathbf{F}_{0}\left(\nu_{0}\right)=-\left[c / 3 \chi_{0}\left(\nu_{0}\right)\right] \nabla E_{0}\left(\nu_{0}\right) .
$$

Using this expression in (95.82) we obtain the nonequilibrium-diffusionlimit monochromatic radiation-energy equation

$$
\begin{aligned}
\rho \| \frac{D}{D t}\left[\frac{E_{0}\left(\nu_{0}\right)}{\rho}\right]+\frac{1}{3}\left\{E_{0}\left(\nu_{0}\right)-\frac{\partial}{\partial \nu_{0}}\left[\nu_{0} E_{0}\left(\nu_{0}\right)\right]\right\} \frac{D}{D t}\left(\frac{1}{\rho}\right) \rrbracket \\
=\nabla \cdot\left[\frac{c}{3 \chi_{0}\left(\nu_{0}\right)} \nabla E_{0}\left(\nu_{0}\right)\right]+\kappa_{0}\left(\nu_{0}\right)\left[4 \pi B\left(\nu_{0}, T\right)-c E_{0}\left(\nu_{0}\right)\right] .
\end{aligned}
$$

Here we have assumed that $\chi_{0}$ and $\eta_{0}$ are given by (77.7).

Integrating over all frequencies we obtain the total flux

$$
\mathbf{F}_{0}=-(c / 3 \bar{\chi}) \nabla E_{0},
$$

and the nonequilibrium radiation diffusion equation

$$
\rho\left[\frac{D}{D t}\left(\frac{E_{0}}{\rho}\right)+\frac{1}{3} E_{0} \frac{D}{D t}\left(\frac{1}{\rho}\right)\right]=\nabla \cdot\left(\frac{c}{3 \bar{\chi}} \nabla E_{0}\right)+c\left(\kappa_{P} a_{R} T^{4}-\kappa_{E} E_{0}\right),
$$

\footnotetext{
* What we call "equilibrium diffusion" is called radiation heat conduction by some authors (Z1, 151-153), and "radiation diffusion" by others. What we call "nonequilibrium diffusion" is also sometimes called "radiation diflusion" (Z1, 154-156) (which can lead to confusion) or the "Eddington approximation" (which is imprecise because one can invoke isotropy, $P=\frac{1}{3} E$, without dropping the $\partial F / \partial t$ term, which destroys the wavelike character of the radiation moment equations in transparent material and forces them to yield a diffusion equation). We recommend the terminology used here because it is descriptive and specific.
} 
an expression first derived by Castor (C3). Here $\bar{\chi}$ is defined by

$$
\nabla E_{0} / \bar{\chi} \equiv \int_{0}^{\infty}\left[\nabla E_{0}\left(\nu_{0}\right) / \chi_{0}\left(\nu_{0}\right)\right] d \nu_{0}
$$

[cf. (82.22)], taking different values along different coordinate axes if necessary; $\kappa_{P}$ is the Planck mean; and $\kappa_{E}$ is the absorption mean [cf. $(82.30)]$

$$
\kappa_{E} \equiv \int_{0}^{\infty} e\left(\nu_{0}\right) \kappa_{0}\left(\nu_{0}\right) d \nu_{0}
$$

where $e\left(\nu_{0}\right) \equiv E_{0}\left(\nu_{0}\right) / E_{0}$ is the radiation energy spectral profile in the comoving frame.

Equations (97.69) and (97.70) are extensions of first-order equilibrium diffusion theory. One can make similar extensions of the second-order theory $(\mathbf{H 2})$; in essence one replaces $a_{R} T^{4}$ with $E_{0}$ in the expressions for $\mu_{\mathrm{R}}$ and $\mathrm{P}_{0}$.

To emphasize the similarity of $(97.70)$ to the equilibrium diffusion equation (97.7) we parameterize the total radiation energy density in terms of a radiation temperature $T_{R}$, which in general is distinct from the material temperature $T$. Thus, if we define

$$
a_{\mathrm{R}} T_{R}^{4} \equiv E_{0}
$$

equation $(97.70)$ becomes

$$
\rho\left[\frac{D}{D t}\left(\frac{a_{R} T_{R}^{4}}{\rho}\right)+\frac{1}{3} a_{R} T_{R}^{4} \frac{D}{D t}\left(\frac{1}{\rho}\right)\right]=\nabla \cdot\left(\frac{4}{3} \frac{a_{R} c T_{R}^{3}}{\bar{\chi}} \nabla T_{R}\right)+a_{R} c\left(\kappa_{P} T^{4}-\kappa_{E} T_{R}^{4}\right) .
$$

We stress that $T_{R}$ is only a parameter describing the total radiation energy density, and that $e\left(\nu_{0}\right)$ need not be Planckian at $T_{R}$ (although it may be useful to assume that it is-see below). In this two-temperature description we must determine two variables, $T$ and $T_{R}$, hence must solve (97.74) along with the material energy equation

$$
\rho\left[\frac{D e}{D t}+p \frac{D}{D t}\left(\frac{1}{\rho}\right)\right]=a_{R} c\left(\kappa_{E} T_{R}^{4}-\kappa_{P} T^{4}\right)+\rho \varepsilon .
$$

Discrete versions of (97.74) and (97.75) are to be solved simultaneously for $T^{n+1}$ and $T_{R}^{n+1}$ at $t^{n+1}$. Both equations are linearized around current estimates $T^{*}$ and $T_{R}^{*}$; for one-dimensional flows one obtains a block tridiagonal system with $2 \times 2$ blocks. This system is solved by Gaussian elimination, and the linearization procedure is iterated to convergence.

Thus far we have assumed that the coefficients $\bar{\chi}$ and $\kappa_{E}$ are given. In grey material, $\kappa_{\mathrm{P}} \equiv \kappa_{E} \equiv \kappa$ and $\bar{\chi} \equiv \kappa+\sigma$. For nongrey material the simplest approximation is to set, by analogy with equilibrium diffusion, $\bar{\chi}=\chi_{R}$ and $\kappa_{\mathrm{E}}=\kappa_{p}$, with all opacities evaluated at the material temperature. This 
approach assumes that the radiation field is not strongly out of equilibrium with the material. A simple alternative is to take the two-temperature description literally, and assume that the spectral distribution of $E_{0}\left(\nu_{0}\right)$ is actually $B\left(v_{0}, T_{R}\right)$. Then, following $(\mathbf{F 3})$, we can define the two-temperature mean opacities

and

$$
\kappa_{E}\left(T, T_{R}\right) \equiv \int_{0}^{\infty} \kappa_{0}\left(\nu_{0}, T\right) B\left(\nu_{0}, T_{R}\right) d \nu_{0} /\left(a_{R} c T_{R}^{4} / 4 \pi\right),
$$

$$
\bar{\chi}\left(T, T_{R}\right) \equiv\left[\int_{0}^{\infty} \frac{1}{\chi_{0}\left(\nu_{0}, T\right)}\left(\frac{\partial B}{\partial T}\right)_{T_{R}} d \nu_{0} /\left(a_{R} c T_{R}^{3} / \pi\right)\right]^{-1} .
$$

This approach accounts approximately for the nonequilibrium character of the radiation field, and reduces to standard equilibrium diffusion when $T_{R}=T$.

To improve on the schemes described above we must determine the actual spectrum $e\left(\nu_{0}\right)$ by solving the monochromatic diffusion equation (97.68). This equation is complicated mathematically by the frequency derivative, which accounts for the varying Doppler shifts experienced by photons as they travel through moving material. Nonetheless, this term has been written in conservative form, and vanishes when (97.68) is integrated over frequency. One might therefore argue that we could simply drop the frequency derivative, knowing that the correct total energy density would still be obtained. The fallacy of this argument is revealed by a thought experiment devised by Buchler (B3).

Consider an adiabatic enclosure containing an extremely opaque, homogeneous medium (which implies $\mathbf{F} \equiv 0$ ), whose sole opacity is Thomson scattering $(\kappa \equiv 0)$. Then, without the frequency derivative, (97.68) reduces to

$$
\frac{D \ln E_{0}\left(\nu_{0}\right)}{D t}-\frac{4}{3} \frac{D \ln \rho}{D t}=0
$$

which predicts that

$$
E_{0}\left(\nu_{0}\right) \propto V^{-4 / 3} .
$$

This result is correct for the total energy density in an adiabatic enclosure [cf. (69.71)], but not for the monochromatic energy density. Indeed, the fact that $E \propto V^{-4 / 3}$ in equilibrium implies that $T \propto V^{-1 / 3}$, so that the spectral distribution $B\left(\nu_{0}, T\right)$ must change when the enclosure contracts or expands. We will obtain the correct radiation energy spectral profile only if we retain the frequency derivative in (97.68). This term accounts for the progressive redshift (blueshift) of radiation undergoing adiabatic expansion (compression). Moreover, in the nonequilibrium case it allows redistribution of radiation over frequency to produce a non-Planckian spectrum, which may be of critical importance, for example when energy cascades from the spectral peak into a high-energy tail in material undergoing 
compression. In short, the $(\partial / \partial \nu)$ term must be retained in order to obtain the correct spectral distribution $e\left(\nu_{0}\right)$ (which is the whole point of doing a frequency-dependent calculation!).

Introducing frequency groups (or ODF pickets) as in $\$ 82$, in onedimensional flows we must solve $G$ diffusion equations

$$
\begin{aligned}
\frac{D}{D t}\left(\frac{E_{g}}{\rho}\right)+\frac{1}{3}\left[E_{g}-\right. & \left.\Delta_{g}\left(\nu E_{\nu}\right)\right] \frac{D}{D t}\left(\frac{1}{\rho}\right) \\
& =\frac{\partial}{\partial M_{r}}\left[\frac{c\left(4 \pi r^{2}\right)^{2}}{3\left(\bar{X}_{g} / \rho\right)} \frac{\partial E_{g}}{\partial M_{r}}\right]+4 \pi\left(\frac{\kappa_{P, g}}{\rho}\right) B_{g}-c\left(\frac{\bar{\kappa}_{g}}{\rho}\right) E_{g}
\end{aligned}
$$

simultaneously with the material energy equation

$$
\frac{D e}{D t}+p \frac{D}{D t}\left(\frac{1}{\rho}\right)=\sum_{\mathrm{g}}\left[c\left(\frac{\bar{\kappa}_{\mathrm{g}}}{\rho}\right) E_{\mathrm{g}}-4 \pi\left(\frac{\kappa_{P, g}}{\rho}\right) B_{\mathrm{g}}\right]
$$

where

$$
E_{\mathrm{g}}=\int_{\nu^{\prime} \mathrm{s}}^{\nu_{\mathrm{s}+1}} E_{0}\left(\nu_{0}\right) d \nu_{0}
$$

Here $\kappa_{P, g}$ is the group Planck mean defined by (82.42), and $\bar{\kappa}_{\mathrm{g}}$ and $\bar{\chi}_{\mathrm{g}}$ are, respectively, appropriate direct and harmonic averages of the opacity within the group.

There are several possible choices for $\bar{\kappa}_{\mathrm{g}}$ and $\bar{\chi}_{\mathrm{g}}$ (cf. $\$ 82$ ). For example, one might choose $\bar{\kappa}_{\mathrm{g}}=\kappa_{\mathrm{P}_{\mathrm{g}}}(T)$ and $\bar{\chi}_{\mathrm{g}}=\chi_{\mathrm{R}, \mathrm{g}}(T)$ computed at the material temperature $T$. Or, generalizing (97.76) and (97.77), one can introduce two-temperature group means $(\mathbf{F 3})$ and set $\bar{\kappa}_{\mathrm{g}}=\kappa_{\mathrm{P}, \mathrm{g}}\left(T, T_{R}\right)$ and $\bar{\chi}_{\mathrm{g}}=$ $\chi_{R}\left(T, T_{R}\right)$ where

$$
\kappa_{P, \mathrm{~g}}\left(T, T_{R}\right) \equiv \int_{\nu_{\mathrm{R}}}^{\nu_{\mathrm{g} \cdots+1}} \kappa_{0}\left(\nu_{0}, T\right) B\left(\nu_{0}, T_{R}\right) d \nu_{0} / B_{\mathrm{g}}
$$

and

$$
\chi_{R, g}\left(T, T_{R}\right) \equiv\left[\int_{\nu_{g}}^{\nu_{\mathrm{k}+1}} \frac{1}{\chi_{0}\left(\nu_{0}, T\right)}\left(\frac{\partial B_{v}}{\partial T}\right)_{T_{R}} d \nu_{0} / \int_{\nu_{\mathrm{g}}}^{\nu_{\mathrm{s}+1}}\left(\frac{\partial B_{\nu}}{\partial T}\right)_{T_{R}} d \nu_{0}\right]^{-1}
$$

In either case $\kappa_{P, g}$ in the emission term remains a function of $T$ only. Another possibility is to use an ODF, in which case $\bar{\kappa}_{\mathrm{g}}=\kappa_{\text {picket }}(T)$ and $\bar{\chi}_{\mathrm{g}}=\chi_{\text {picket }}(T)$, which are constants within each picket; we then also use $\kappa_{\text {picket }}$ for $\kappa_{P, g}$ in the emission term. For expository ease we will use the generic notation $\bar{\kappa}_{\mathrm{g}}$ and $\bar{\chi}_{\mathrm{g}}$ to represent any of the choices just described.

The term $\Delta_{g}\left(\nu E_{\nu}\right)$ in $(97.80)$ denotes the integral of $\partial\left[\nu_{0} E_{0}\left(\nu_{0}\right)\right] / \partial \nu_{0}$ over group g. We use upstream differencing, remembering that photons are redshifted (blueshifted) if the material expands (is compressed). hence for expansion we couple group $g$ to $g+1$ and write

$$
\Delta_{g}\left(\nu E_{\nu}\right)=\left(\nu E_{\nu}\right)_{g+1}-\left(\nu E_{\nu}\right)_{g}=\nu_{g+(3 / 2)}\left(E_{g+1} / \Delta \nu_{g+1}\right)-\nu_{g+(1 / 2)}\left(E_{g} / \Delta v_{g}\right)
$$


and for compression we couple group $g$ to group $g-1$ :

$$
\Delta_{\mathrm{g}}\left(\nu E_{\nu}\right)=\nu_{\mathrm{g}+(1 / 2)}\left(E_{\mathrm{g}} / \Delta \nu_{\mathrm{g}}\right)-\nu_{\mathrm{g}-(1 / 2)}\left(E_{\mathrm{g}-1} / \Delta \nu_{\mathrm{g}-1}\right)
$$

Here $\nu_{\mathrm{g}+(1 / 2)} \equiv \frac{1}{2}\left(\nu_{\mathrm{g}}+\nu_{\mathrm{g}+1}\right)$, and the divisor $\Delta \nu_{\mathrm{g}} \equiv\left(\nu_{\mathrm{g}+1}-\nu_{\mathrm{g}}\right)$ enters to convert $E_{\mathrm{g}}$, an integral over the frequency band, back to a spectral density $E_{\nu}$. When the discrete form of $(97.80)$ is summed over all groups, the $\Delta\left(\nu E_{\nu}\right)$ terms telescope, and their sum vanishes identically.

The multigroup diffusion equations and the material energy equation are discretized in space and time in the usual way. If one uses two-temperature opacities, it is convenient to adjoin the definition

$$
\sum_{\mathrm{g}} E_{\mathrm{g}}=a_{R} T_{R}^{4}
$$

at each depth point. Assuming that all material properties are $f(\rho, T)$ or $f\left(\rho, T, T_{R}\right)$, and that $\rho^{n+1}$ is known from the explicit hydrodynamics, the goal is to determine the solution vectors

$$
\psi_{d} \equiv\left(E_{1}, \ldots, E_{G}, T, T_{R}\right)_{d}^{n+1}, \quad(d=1, \ldots, D)
$$

of the nonlinear system.

Consider first a direct solution of the problem. We linearize all equations around current values $\boldsymbol{\psi}_{d}^{*}$; material properties are linearized in terms of $\delta T$ (and, if appropriate, $\delta T_{R}$ ). The resulting linear system in $\left(\delta E_{1}, \ldots, \delta E_{G}, \delta T, \delta T_{R}\right)$ is block tridiagonal, and can be solved using the Feautrier technique described in $\$ 88$ and iterated to convergence. The total computational effort scales as $c D(G+2)^{3}$; the solution is thus costly for a large number of frequency groups, and an alternative procedure may be preferable.

An efficient iteration scheme is provided by the multifrequency/grey method, which was developed in the VERA code [see (F4) and $\$ \$ 98$ and 99]. Here we break the solution into two parts, each of which can be done relatively cheaply. In the first step, we assume that we know the radiation energy spectral profile $e_{\mathrm{g}} \equiv E_{\mathrm{g}} / E_{0}$. We then compute

and

$$
\kappa_{E} \equiv \sum_{\mathrm{g}} e_{\mathrm{g}} \bar{\kappa}_{\mathrm{g}} \equiv k_{E} \kappa_{\mathrm{P}}
$$

$$
(1 / \bar{\chi}) \equiv \sum_{\mathrm{g}}\left(1 / \bar{\chi}_{\mathrm{g}}\right)\left(\partial E_{\mathrm{g}} / \partial M_{r}\right) /\left(\partial E_{0} / \partial M_{r}\right) \equiv k_{F} \chi_{R},
$$

and use these means in the integrated diffusion equation (97.74) and the material energy equation (97.75). We linearize these equations (holding the ratios $k_{E}$ and $k_{F}$ fixed), solve for $\delta T$ and $\delta T_{R}$, and update $T$ and $T_{R}$ at all depths; this step requires only $c D(2)^{3}$ operations. In the second step, we assume that we know the run of $T$ and $T_{R}$. We can then solve the multigroup equations (97.80) for all $E_{\mathrm{g}}$ 's using an "inner iteration" procedure. To avoid coupling among the equations, we put the $\Delta_{g}\left(\nu E_{\nu}\right)$ terms on the right-hand side along with the source term $B_{\mathrm{g}}$. To start, we use the old 
$e_{\mathrm{g}}$ scaled to the current value of $E_{0}$ to estimate the $\Delta_{\mathrm{g}}$ 's. We then have $G$ independent tridiagonal systems of order $D$, which can be solved in parallel with a computational effort that scales as cDG. We then update the $\Delta_{\mathrm{g}}$ 's on the right-hand side, and iterate to convergence, thus determining $e_{\mathrm{g}}$. We then return to the first step to compute more accurate values of $T$ and $T_{R}$, and carry this "outer iteration" to convergence.

The multifrequency/grey method is efficient because in the first step even a rough estimate of the spectral distribution yields reasonable values for $\kappa_{E}$ and $\bar{\chi}$, hence for $T$ and $T_{R}$. Similarly, in the second step the computed spectral distribution will be reasonably accurate even if $T$ and $T_{R}$ (hence $e$ and $E_{0}$ ) contain local errors.

Before leaving nonequilibrium diffusion theory, it is worthwhile to critique earlier formulations [e.g., (C2) and (F3)] which, unfortunately, are not physically consistent. In essence, these analyses start from the lab-frame equations (93.10) and (93.11), assume that $P^{i i}=\frac{1}{3} E \delta^{i j}$, and drop all $\mathrm{O}(v / c)$ terms on the right-hand side, obtaining an energy equation of the form

$$
(\partial E / \partial t)=\kappa(4 \pi B-c E)+\nabla \cdot[(c / 3 \chi) \nabla E] .
$$

The problem is that $(97.90)$ is not correct in either the Lagrangean or Eulerian frame. Although the right-hand side of (97.90) looks like the right-hand side of the Lagrangean equation (97.70) (if we ignore the distinction between $E$ and $\left.E_{0}\right),(97.90)$ is nevertheless not Lagrangean because on the left-hand side the distinction between $(\partial E / \partial t)$ and $\rho[D(E / \rho) / D t]$ is not made, and the rate of work done by radiation pressure, $\frac{1}{3} \rho E[D(1 / \rho) / D t]$, is missing. On the other hand (97.90) is not a correct Eulerian equation because on comparing with (93.10) we see that the right-hand side lacks a term $c^{-1} \chi \mathbf{v} \cdot \mathbf{F}$ equal to the rate of work done by radiation forces on the material, and furthermore that in the flux divergence the comoving-frame flux has been used instead of the lab-frame flux, thus omitting the dominant term $\frac{4}{3} E_{0} \mathbf{v}$ that discriminates $\mathbf{F}$ (Eulerian) from $\mathbf{F}_{0}$ (Lagrangean).

Indeed, the derivation of the correct Eulerian nonequilibrium diffusion equation is a bit tricky. Not only must we discriminate between $\mathbf{F}$ and $\mathbf{F}_{0}$, but we also must be careful how we relate the inertial-frame energy density $E$ to the parameter $T_{R}$. If we naively write $E=\frac{1}{3} a_{R} T_{R}^{4}$, the radiation-energy and gas-energy equations contain extra terms and do not reduce correctly to their Lagrangean counterparts (although the result for the radiating fluid-radiation plus material-is correct). Instead, we must define $T_{R}$ by (97.73); then (91.16) implies that

$$
E=a_{R} T_{R}^{4}-\left(8 a_{R} T_{R}^{3} / 3 c \chi_{R}\right) \mathbf{v} \cdot \nabla T_{R}
$$

The second term in (97.91) is important only when we compute the net absorption-emission. Using (97.91) and (93.14) in (93.10), we find that the Eulerian nonequilibrium-diffusion radiation energy equation correct to 
$\mathrm{O}(v / c)$ is

$$
\begin{array}{r}
\left(a_{R} T_{R}^{4}\right)_{, t}=a_{R} c\left(\kappa_{\mathrm{P}} T^{4}-\kappa_{E} T_{R}^{4}\right)+\nabla \cdot\left[\left(4 a_{R} c T_{R}^{3} / 3 \chi_{R}\right) \nabla T_{R}-\frac{4}{3} a_{R} T_{R}^{4} \mathbf{v}\right] \\
+\frac{4}{3} a_{R} T_{R}^{3} \mathbf{v} \cdot \nabla T_{R},
\end{array}
$$

which reduces to the more revealing form

$$
\begin{aligned}
\left(a_{\mathrm{R}} T_{R}^{4}\right)_{\mathrm{t}}+\boldsymbol{\nabla} \cdot\left(a_{\mathrm{R}} T_{\mathrm{R}}^{4} \mathbf{v}\right)+\frac{1}{3} a_{R} T_{R}^{4} \boldsymbol{\nabla} \cdot \mathbf{v} & \\
= & a_{R} c\left(\kappa_{\mathrm{P}} T^{4}-\kappa_{E} T_{R}^{4}\right)+\nabla \cdot\left[\left(4 a_{R} c T_{R}^{3} / 3 \chi_{R}\right) \nabla T_{R}\right]
\end{aligned}
$$

which is identical to (97.74). Similarly, using (97.92) in (94.20) we find that the Eulerian gas-energy equation, correct to $\mathrm{O}(v / c)$, in the nonequilibrium diffusion limit is

$$
(\rho e)_{, t}+\boldsymbol{\nabla} \cdot(\rho e \mathbf{v})+p \boldsymbol{\nabla} \cdot \mathbf{v}=a_{R} c\left(\kappa_{E} T_{R}^{4}-\kappa_{P} T^{4}\right)+\rho \varepsilon,
$$

which is identical to (97.75).

In summary, (97.90) is unsatisfactory because either a term equal to four times the radiation work term is omitted if it is used as a Lagrangean equation, or the wrong flux is computed and a term equal to the rate of work done by radiation is omitted if it is used as an Eulerian equation.

THE PROBLEM OF FLUX LIMITING IN DIFFUSION THEORY

Inasmuch as the basic assumption of diffusion theory is that $\lambda_{p} \ll l$, there is no reason to expect the time-dependent radiation diffusion equation to yield accurate results in transparent media. Nevertheless, because of its simplicity, diffusion theory is often used throughout the flow, in both opaque and transparent regions; typically one then finds (B1), (C2) too large an energy transport in the optically thin material. Moreover, diffusion theory usually gives a serious overestimate of the energy deposited by a radiation front penetrating into cold material, particularly at early times. Nonequilibrium diffusion generally gives better results than equilibrium diffusion, but both are significantly in error.

In the most extreme cases, the flux out of an optically thin zone predicted by diffusion theory may exceed the energy density times the velocity of light,

$$
\left|\mathbf{F}_{\text {diffusion }}\right|>c E
$$

implying that the effective speed of energy propagation,

$$
V_{E} \equiv|\mathbf{F}| / E=\left|\frac{1}{3} c \lambda_{\mathrm{p}} \nabla E\right| / E \sim c\left(\lambda_{\mathrm{p}} / 3 l\right)
$$

exceeds the velocity of light.

Both of these results are physically absurd, and clearly reflect a breakdown of the theory. The root of the problem is that the diffusion equation tacitly assumes that photons always travel a distance of the order of $\lambda_{\mathrm{p}}$, even if $\lambda_{p}$ exceeds the free-flight distance $c \Delta t$ corresponding to the timestep $\Delta t$. To follow a radiation front we choose $\Delta t \sim \Delta x / c$, hence we can expect diffusion theory to break down in regions where $\lambda_{p}>\Delta x$. The 
problem should come as no surprise, for it is well known that the linear diffusion equation has a formally infinite signal speed (M16, 862-865), whereas the signal speed for transport of radiation in transparent material is limited to the speed of light $(\$ 81)$.

One way to overcome the problem is to use a flux limiter, first suggested by J. R. Wilson (unpublished); see also (W4). The idea is to alter the diffusion-theory formula for the flux in such a way as to yield the standard result in the high-opacity limit, while simulating free streaming in transparent regions. For example, we might use an expression of the form

$$
\mathbf{F}=-c \nabla E /\left[\left(3 / \lambda_{p}\right)+|\nabla E| / E\right]
$$

which yields $\mathbf{F}_{\text {diffusion }}$ when $\lambda_{p} / l \ll 1$, and limits to

$$
\mathbf{F}=c \mathbf{E n}
$$

in transparent regions; here $\mathbf{n}$ is a unit vector opposite to $\boldsymbol{\nabla} E$ (i.e., down the gradient). Equation (97.97) is only illustrative; numerous other expressions have been proposed (K2), (L3), (L4), the one most often used in astrophysics appearing in (A1).

While flux-limited diffusion has been widely used, this approach provides only an ad hoc "fixup" of generally unknown accuracy; it gives the correct limits, and has qualitatively correct behavior in between, but it could be quantitatively wrong (perhaps seriously) in the intermediate regime. Fundamentally the flux-limiting problem results from dropping the time derivative from the radiation momentum equation, which precludes recovery of the wave-equation character of the coupled radiation energy and momentum equations in the optically thin limit. Indeed, dimensional analysis suggests that on a radiation-flow time scale $c^{-2}(\partial F / \partial t)$ is $\mathrm{O}\left(\lambda_{p} / l\right)$ relative to $\chi F / c$, and will dominate the solution for a radiation front in transparent material.

If we retain the time derivative in the radiation momentum equation, we can, in fact, recover the wave equation in transparent material, and the solution is automatically flux limited (M10). We examine this assertion briefly here to motivate the developments of $\$ \$ 98$ and 99 . To make the point while avoiding unnecessary complications, we consider timedependent transport in planar, static material. The radiation energy and momentum equations are

$$
(\partial E / \partial t)+(\partial F / \partial z)=\kappa(4 \pi B-c E)
$$

and

$$
c^{-2}(\partial F / \partial t)+[\partial(f E) / \partial z]=-(\chi / c) F
$$

where $f$ is the variable Eddington factor. We first replace the time derivative in $(97.100)$ by a finite difference, while leaving the spatial derivative in continuous form; for stability we use a fully implicit scheme, 
which gives

where

$$
F^{n+1}=\frac{-c}{\gamma+\chi^{n+1}} \frac{\partial\left(f^{n+1} E^{n+1}\right)}{\partial z}+\frac{\gamma}{\gamma+\chi^{n+1}} F^{n}
$$

$$
\gamma \equiv 1 / c \Delta t
$$

Equation (97.101) provides an analytical expression for the flux, which can be used in (97.99); but let us first examine its physical content.

Define $\lambda$ such that

$$
\lambda^{-1} \equiv \gamma+\chi=(c \Delta t)^{-1}+\lambda_{p}^{-1}=\lambda_{t}^{-1}+\lambda_{p}^{-1} .
$$

Clearly the effective mean free path $\lambda$ is the harmonic mean of the optical mean free path $\lambda_{p}$ and the free-flight distance $\lambda_{t}$ through which a photon can travel in a timestep $\Delta t$. We then rewrite (97.101) as

$$
F^{n+1}=-c \lambda^{n+1}\left[\partial\left(f^{n+1} E^{n+1}\right) / \partial z\right]+\left(\lambda / \lambda_{t}\right) F^{n} .
$$

In opaque material $\chi \gg 1, \lambda_{p} \ll \lambda_{t}$, and $\lambda \rightarrow \lambda_{p}$, while $f \rightarrow \frac{1}{3}$. Equation (97.104) then reduces to the standard diffusion result

$$
F^{n+1}=-\frac{1}{3} c \lambda_{p}^{n+1}\left(\partial E^{n+1} / \partial z\right)
$$

In transparent material $\chi \rightarrow 0, \lambda_{p} \rightarrow \infty$, hence $\lambda \rightarrow \lambda_{1}$, which shows that (97.103) correctly limits the effective mean free path to the photon flight distance instead of allowing it to become arbitrarily large. In this regime $f \rightarrow 1, F \rightarrow c E$, and $(97.104)$ reduces to

$$
F^{n+1}=F^{n}-\lambda_{1}\left(\partial F^{n+1} / \partial z\right)
$$

which makes the physically correct statement that in the optically thin limit a change in the local value of the flux results from information communicated about the flux gradient within a photon free-flight distance $\lambda_{t}$.

If we now replace the time derivative in the energy equation with a backwards time difference and eliminate the flux via (97.104), we obtain the combined moment equation

$$
\begin{aligned}
&\left(\gamma+\kappa^{n+1}\right) E^{n+1}-\frac{\partial}{\partial z}\left[\lambda^{n+1} \frac{\partial\left(f^{n+1} E^{n+1}\right)}{\partial z}\right] \\
&=\left(\frac{4 \pi}{c}\right) \kappa^{n+1} B^{n+1}+\gamma E^{n}-\left(\frac{\gamma}{c}\right) \frac{\partial}{\partial z}\left(\lambda^{n+1} F^{n}\right)
\end{aligned}
$$

The mathematical structure of (97.107) is similar to the nonequilibrium diffusion equation (97.70); its physical content, however, is much larger. In the limit of high opacity and/or long timesteps, $(\kappa / \gamma) \gg 1$, and (97.107) reduces to

$$
\frac{1}{3} \frac{\partial}{\partial z}\left(\lambda_{p}^{n+1} \frac{\partial E^{n+1}}{\partial z}\right)=\kappa^{n+1}\left[E^{n+1}-\left(\frac{4 \pi}{c}\right) B^{n+1}\right]
$$


which is a quasi-static nonequilibrium diffusion equation at the advanced time level. In the limit of low opacity and/or short timesteps, $(\kappa / \gamma) \ll 1$, and, setting $f=1$, we find

$$
E^{n+1}-(c \Delta t)^{2}\left(\partial^{2} E^{n+1} / \partial z^{2}\right)=E^{n}-(\gamma c)^{-1}\left(\partial F^{n} / \partial z\right)=2 E^{n}-E^{n-1},
$$

where the second equality follows from a backward Euler representation of (97.99) at $t^{n}$ for $\kappa=0$. Regrouping terms we have

$$
c^{2}\left(\partial^{2} E^{n+1} / \partial z^{2}\right)=\left(E^{n+1}-2 E^{n}+E^{n-1}\right) / \Delta t^{2} \approx\left(\partial^{2} E / \partial t^{2}\right)^{n},
$$

which is an approximation to the wave equation; the miscentering of the time derivative results from use of fully implicit difference formulae in both moment equations. If we were to use $f=\frac{1}{3}$ in the transparent limit we would still obtain the wave equation, but with a propagation speed of only $c / \sqrt{3}$ instead of $c$, a result that also follows rigorously from (97.99) and (97.100) when $\kappa=\chi \equiv 0$ and $f=\frac{1}{3}$. Thus it is essential to use accurate Eddington factors in order to obtain the correct propagation speed.

In summary, if we solve the full time-dependent radiation moment equations we recover both the diffusion and wave limits, and avoid the problem of flux limiting, which is a mere artifact of the approximations inherent in the diffusion equation. Furthermore, we must use accurate Eddington factors if we are to recover the correct streaming limit. Let us therefore now consider how to solve the full radiation transport problem in moving media.

\section{Transport Solution in the Comoving Frame}

In this section we discuss methods for solving the Lagrangean equations of radiation hydrodynamics in the transport regime. Because the complete set of equations is complicated, we tailor the discussion to situations of astrophysical interest. As before we consider only one-dimensional spherically symmetric flows of a single material. We will emphasize fluid-flow time scales, but write equations that generally behave correctly on radiation-flow time scales as well. To close the system of moment equations we use variable Eddington factors; these are to be evaluated in a subsidiary angle-frequency-dependent formal solution. Similarly, the spectral distributions required to form mean absorption coefficients for the radiation energy and momentum equations are to be obtained from a subsidiary solution of multigroup equations.

RADIATION ENERGY AND MOMENTUM EQUATIONS

For economy of notation we omit the affix " 0 " with the understanding that in this section all physical variables are measured in the comoving frame. As discussed in $\$ 95$, we retain all $\mathrm{O}(v / c)$ terms in the radiation energy equation; in the radiation momentum equation we drop them all except the 
$(D / D t)$ term, which is kept to assure flux limiting, as discussed in $\$ 97$. Thus the radiation equations to be solved are the monochromatic radiation energy equation

$$
\begin{aligned}
\frac{D}{D t}\left(\frac{E_{v}}{\rho}\right)+\left[f_{\nu} \frac{D}{D t}\left(\frac{1}{\rho}\right)-\left(3 f_{\nu}-1\right) \frac{v}{\rho r}\right] & E_{\nu}-\frac{\partial}{\partial \nu}\left\{\left[f_{\nu} \frac{D}{D t}\left(\frac{1}{\rho}\right)-\left(3 f_{\nu}-1\right) \frac{v}{\rho r}\right] \nu E_{\nu}\right\} \\
& =\frac{\kappa_{\nu}}{\rho}\left(4 \pi B_{\nu}-c E_{\nu}\right)-\frac{\partial\left(4 \pi r^{2} F_{\nu}\right)}{\partial M_{r}},
\end{aligned}
$$

and the monochromatic radiation momentum equation

$$
\frac{1}{c^{2}} \frac{D F_{\nu}}{D t}+4 \pi r^{2} \rho \frac{\partial\left(f_{\nu} E_{\nu}\right)}{\partial M_{r}}+\frac{\left(3 f_{\nu}-1\right)}{r} E_{\nu}=-\frac{\chi_{\nu} F_{\nu}}{c} .
$$

As in $\$ 83$ we define the sphericity factor

$$
\ln q_{v} \equiv \int_{r_{c}}^{r}\left[\left(3 f_{\nu}-1\right) / f_{\nu} r^{\prime}\right] d r^{\prime}
$$

which allows us to rewrite (98.2) as

$$
\frac{1}{c^{2}} \frac{D F_{v}}{D t}+\frac{4 \pi r^{2} \rho}{q_{v}} \frac{\partial\left(f_{v} q_{v} E_{v}\right)}{\partial M_{r}}=-\frac{\chi_{v} F_{v}}{c} .
$$

Integrating (98.1) over frequency we obtain the radiation energy equation

$$
\frac{D}{D t}\left(\frac{E}{\rho}\right)+\left[f \frac{D}{D t}\left(\frac{1}{\rho}\right)-(3 f-1) \frac{v}{\rho r}\right] E=\frac{1}{\rho}\left(4 \pi \kappa_{P} B-c \kappa_{E} E\right)-\frac{\partial\left(4 \pi r^{2} F\right)}{\partial M_{r}} .
$$

Here

$$
f \equiv \int_{0}^{\infty} f_{\nu} e_{\nu} d \nu
$$

and

$$
\kappa_{E} \equiv \int_{0}^{\infty} \kappa_{\nu} e_{\nu} d \nu
$$

where the radiation energy spectral profile $e_{\nu} \equiv \mathrm{E}_{\nu} / \mathrm{E}$ is presumed known. Similarly, integrating (98.2) over frequency we obtain the radiation momentum equation

$$
\frac{1}{c^{2}} \frac{D F}{D t}+\frac{4 \pi r^{2} \rho}{q} \frac{\partial(f q E)}{\partial M_{r}}=-\cdots \frac{\chi_{F} F}{c},
$$

where now

$$
\ln q \equiv \int_{r_{c}}^{r}\left[(3 f-1) / f r^{\prime}\right] d r^{\prime}
$$

and

$$
\chi_{F} \equiv \int_{0}^{\infty} \chi_{\nu} f_{\nu} d \nu
$$

Again the radiation flux spectral profile $f_{\nu} \equiv F_{\nu} / F$ is assumed known. 
We can use (98.5) and (98.8) as a coupled system on an interleaved grid with $E$ defined at cell centers and $F$ defined on cell boundaries. Alternatively we can use (98.8) to eliminate $F$ from the radiation energy equation, thereby producing a combined moment equation for $E$. To obtain such an equation we replace the time derivative in (98.8) by a finite difference while leaving the spatial operator in continuous form. For stability we use a backwards difference, obtaining

$$
F=-\frac{4 \pi c r^{2} \rho \lambda}{q} \frac{\partial(f q E)}{\partial M_{r}}+\left(\frac{\lambda}{\lambda_{l}}\right) F^{n}
$$

where $\lambda$ is the effective mean free path defined in (97.103), and $\lambda_{t} \equiv c \Delta t$. In (98.11), all variables are evaluated at a time $\Delta t$ beyond some arbitrary reference time $t^{n}$; notice that as $\Delta t \rightarrow 0, \lambda \rightarrow 0,\left(\lambda / \lambda_{t}\right) \rightarrow 1$, and $F \rightarrow F^{n}$. Substituting (98.11) into (98.5) we have

$$
\begin{aligned}
\frac{D}{D t}\left(\frac{E}{\rho}\right) & +\left[f \frac{D}{D t}\left(\frac{1}{\rho}\right)-(3 f-1) \frac{v}{\rho r}\right] E=\frac{1}{\rho}\left(4 \pi \kappa_{p} B-c \kappa_{E} E\right) \\
& +\frac{\partial}{\partial M_{r}}\left[\frac{\left(4 \pi r^{2}\right)^{2} c \rho \lambda}{q} \frac{\partial(f q E)}{\partial M_{r}}\right]-\frac{\partial}{\partial M_{r}}\left[4 \pi r^{2}\left(\frac{\lambda}{\lambda_{t}}\right) F^{n}\right] .
\end{aligned}
$$

If we ignore the time dependence of the radiation momentum equation (equivalent to letting $\lambda_{t} \rightarrow \infty, \lambda \rightarrow \lambda_{p}$ ) we obtain the combined moment equation

$$
\begin{aligned}
\frac{D}{D t}\left(\frac{E}{\rho}\right)+\left[f \frac{D}{D t}\left(\frac{1}{\rho}\right)-(3 f-1)\right. & \left.\frac{v}{\rho r}\right] E \\
& =\frac{1}{\rho}\left(4 \pi \kappa_{P} B-c \kappa_{E} E\right)+\frac{\partial}{\partial M_{r}}\left[\frac{\left(4 \pi r^{2}\right)^{2} c \rho}{q \chi_{F}} \frac{\partial(f q E)}{\partial M_{r}}\right],
\end{aligned}
$$

which was first derived by Castor (C3) (who made the additional approximations that $\kappa_{E}=\kappa_{P}$ and $\chi_{F}=\chi_{R}$ ). This equation can be used in a variety of astrophysical problems [see e.g., (K3)] but (98.12) is preferable if we wish to model phenomena on a radiation-flow time scale. If we set $f \equiv \frac{1}{3}$ and $q \equiv 1$ in (98.13) we recover the nonequilibrium diffusion equation (97.70) or $(97.74)$; if, instead, we set $(D / D t) \equiv 0$ and $v \equiv 0$, we recover (83.65), the combined moment equation for a static medium.

FLUID EQUATIONS

The radiation equations are to be solved simultaneously with the fluid momentum equation

$$
(D v / D t)=-\left(G M_{r} / r^{2}\right)-4 \pi r^{2}\left[\partial(p+Q) / \partial M_{r}\right]+\left(\chi_{F} / \rho c\right) F, \quad(98.14 a)
$$

OI

$$
(D v / D t)=-\left(G M_{r} / r^{2}\right)-4 \pi r^{2}\left\{\left[\partial(p+Q) / \partial M_{r}\right]+(1 / q)\left[\partial(f q E) / \partial M_{r}\right]\right\}
$$


and the material energy equation

$$
(D e / D t)+(p+Q)[D(1 / \rho) / D t]=\varepsilon+\left(c \kappa_{E} E-4 \pi \kappa_{P} B\right) / \rho \equiv \dot{e}
$$

Here $Q$ is the pseudoviscous pressure, and $\varepsilon$ is the rate of thermonuclear energy generation; all material properties are assumed to be known functions of $\rho$ and $T$.

\section{COMPUTATIONAL STRATEGY}

The problem is to solve (98.14) and (98.15) coupled to either (98.5) and (98.8), or to (98.12), as a function of time. As an example we outline the computational procedure in a Lagrangean coordinate system; but we remind the reader that any coordinate grid may be used, with adaptivemesh schemes (T3), (W3) being the preferred choice for many problems.

In an explicit Lagrangean scheme (cf. \$59) we may use either (98.14a) or (98.14b) to advance $v$ from $t^{n-(1 / 2)}$ to $t^{n+(1 / 2)}$ because $p, Q, E, F, f$, and $q$ are all known at $t^{n}$. Equation (98.14a) is essentially exact, whereas (98.14b) omits both time- and velocity-dependent terms in accordance with the analysis of $\$ 96$. The omitted terms are at most $\mathrm{O}(v / c)$, hence their effect on energy balance is at most $\mathrm{O}\left(v^{2} / c^{2}\right)$.

Having obtained $v^{n-(1 / 2)}$ we update radii and densities to $t^{n+1}$. We must then solve (98.14) simultaneously with either (98.12) for $T$ and $E$, or with (98.5) and (98.8) for $T, E$, and $F$ at $t^{n+1}$. Here we assume that $f, q$, $\kappa_{E}$, and $\chi_{F}$ are known at $t^{n+1}$, which implies that we are given $f_{\nu}, q_{\nu}, e_{\nu}$, and $f_{\nu}$ at $t^{n+1}$. But of course these quantities depend on $T^{n+1}$ and $E^{n+1}$, and the whole set must be determined self-consistently.

The computationally most economical method proceeds in three steps, which are iterated to consistency. (1) Given estimates of the geometric factors and spectral profiles we solve the coupled material energy and radiation moment equations for $T^{n+1}, E^{n+1}$, and $F^{n+1}$. (2) Given these values of $T$ and $E$ we evaluate the source-sink terms in the anglefrequency-dependent transfer equation and perform a formal solution for $f_{v}$ and $q_{v}$. Because the Eddington factor is only a ratio of radiation moments and is primarily geometry dependent, reasonable distributions of $T$ and $E$ yield relatively accurate values of $f_{v}$ and $q_{v}$. (3) Using the current estimates of $T^{n+1}, f_{v}$, and $q_{v}$, we solve the monochromatic moment equations to obtain new estimates of the spectral profiles $e_{v}$ and $f_{\nu}$. We then update the frequency-integrated quantities $f$ and $q$, re-evaluate $\chi_{E}$ and $\chi_{F}$, and return to step (1).

Aside from the additional step of updating Eddington factors, the above procedure is identical to the multifrequency/grey method of solving the multigroup diffusion equation. Indeed, if the Eddington factors can be determined reasonably cheaply (perhaps being updated only every few timesteps), little more effort is needed to solve the full transport equations 
than the corresponding diffusion equations. Inasmuch as a transport solution is inherently much more accurate in transparent regions near boundaries, it may be false economy to use a diffusion calculation at all.

DIFTERENCE EQUATIONS

An explicit difference equation for the fluid momentum equation is

$$
\begin{aligned}
& {\left[v_{i}^{n+(1 / 2)}-v_{i}^{n-(1 / 2)}\right] / \Delta t^{n}=-G M_{i} /\left(r_{i}^{n}\right)^{2}} \\
& \quad-4 \pi\left(r_{i}^{n}\right)^{2}\left[p_{i+(1 / 2)}^{n}-p_{i-(1 / 2)}^{n}+Q_{i+(1 / 2)}^{n-(1 / 2)}-Q_{i-(1 / 2)}^{n-(1 / 2)}\right] / \Delta M_{i}+\left(\chi_{i}^{n} / \rho_{i}^{n} c\right) F_{i}^{n}, \\
& \text { or } \begin{aligned}
{\left[v_{i}^{n+(1 / 2)}-\right.} & \left.v_{i}^{n-(1 / 2)}\right] / \Delta t^{n}=-G M_{i} /\left(r_{i}^{n}\right)^{2} \\
& -4 \pi\left(r_{i}^{n}\right)^{2}\left\{p_{i+(1 / 2)}^{n}-p_{i-(1 / 2)}^{n}+Q_{i+\{(1 / 2)}^{n-(1 / 2)}-Q_{i-(1 / 2)}^{n-(1 / 2)}\right. \\
& \left.+\left[f_{i+(1 / 2)}^{n} q_{i+(1 / 2)}^{n} E_{i+(1 / 2)}^{n}-f_{i-(1 / 2)}^{n} q_{i-(1 / 2)}^{n} E_{i-(1 / 2)}^{n}\right] / q_{i}^{n}\right\} / \Delta M_{i} .
\end{aligned}
\end{aligned}
$$

Here $i=2, \ldots, I$, and we have dropped the subscript " $F$ " on $\chi$. In the case of unequal timesteps, one may wish to center both $p_{i+(1 / 2)}$ and $r_{i}$ at $t^{n+\lambda}$, as in (59.64) and (59.87). The pseudoviscous pressure $Q$ is computed according to the prescriptions in $\$ 59$.

At the lower boundary we assume that $v_{1}=f(t)$, a known function of time; for example $v_{1} \equiv 0$ at the center of a star or at the fixed inner boundary of a pulsating envelope. At the upper boundary we assume that the material and pseudoviscous pressures are zero; we further assume that any material outside of $r_{I+-1}$ is optically thin so that radiation quantities are invariant beyond that point. We then have

$$
\begin{aligned}
{\left[v_{I+1}^{n+(1 / 2)}-v_{I+1}^{n-(1 / 2)}\right] / \Delta t^{n}=-G M_{I+1} /\left(r_{I+1}^{n}\right)^{2} } & \\
& +4 \pi\left(r_{I+1}^{n}\right)^{2}\left[p_{I+(1 / 2)}^{n}+Q_{I+(1 / 2)}^{n-(1 / 2)}\right] / \Delta M_{I+1}+\left(\chi_{I+1}^{n} / \rho_{I+1}^{n} c\right) F_{I+1}^{n}
\end{aligned}
$$

or

$$
\begin{aligned}
{\left[v_{I+1}^{n+(1 / 2)}-v_{I+1}^{n-(1 / 2)}\right] / \Delta t^{n} } \\
=-G M_{I+1} /\left(r_{I+1}^{n}\right)^{2}+4 \pi\left(r_{I+1}^{n}\right)^{2}\left\{\left[p_{I+(1 / 2)}^{n}+Q_{I+(1 / 2)}^{n-(1 / 2)}\right] \Delta M_{I-1}\right. \\
\left.\quad+2\left[f_{I+(1 / 2)}^{n} q_{I+(1 / 2)}^{n}-f_{1+1}^{n} q_{I+1}^{n} \dot{f}_{I+1}^{n}\right] E_{I+(1 / 2)}^{n} / q_{I}^{n} \Delta M_{I+\{1 / 2)}\right\}
\end{aligned}
$$

Here $\Delta M_{\mathrm{I}+1} \equiv \frac{1}{2} \Delta M_{I+-(1 / 2)}+\Delta M_{I+(3 / 2)}$, where $\Delta M_{I+(3 / 2)}$ represents any mass assumed to lie outside of $r_{I+1}$, and $\dot{j}_{I+1} \equiv E_{I+1} / E_{I+(1 / 2)}$ is a geometrical factor determined from the formal solution [cf. (83.44)].

Having advanced $v_{i}^{n-(1 / 2)}$ to $v_{i}^{n-(1 / 2)}$ we update shell positions

$$
r_{i}^{n+1}=r_{i}^{n}+v_{i}^{n+(1 / 2)} \Delta t^{n+(1 / 2)}
$$

and densities

$$
\rho_{i+(1 / 2)}^{n+1}=(3 / 4 \pi) \Delta M_{i+(1 / 2)} /\left[\left(r_{i+1}^{n+1}\right)^{3}-\left(r_{i}^{n+1}\right)^{3}\right] .
$$


The material energy equation is represented as

$$
\begin{aligned}
e_{i+(1 / 2)}^{n+1} & -e_{i+(1 / 2)}^{n} \\
& +\left\{\frac{1}{2}\left[p_{i+(1 / 2)}^{n}+p_{i+(1 / 2)}^{n+1}\right]+Q_{i+(1 / 2)}^{n+(1 / 2)}\right\}\left\{\left[1 / \rho_{i+(1 / 2)}^{n+1}\right]-\left[1 / \rho_{i+(1 / 2)}^{n}\right]\right\} \\
= & \Delta t^{n+(1 / 2)}\left[(1-\theta) \dot{e}_{i+(1 / 2)}^{n}+\theta \dot{e}_{i+(1 / 2)}^{n+1}\right], \quad(i=1, \ldots, I),
\end{aligned}
$$

where $\frac{1}{2} \leq \theta \leq 1$, and

$$
\dot{e}_{i+(1 / 2)}^{m} \equiv \varepsilon_{i+(1 / 2)}^{m}+\left[c \kappa_{E, i+(1 / 2)}^{m} E_{i+(1 / 2)}^{m}-4 \pi \kappa_{P, i+(1 / 2)}^{m} B_{i+(1 / 2)}^{m}\right] / \rho_{i+(1 / 2)}^{m} .
$$

To (98.20) we must adjoin difference-equation representations of the radiation energy and momentum equations. We will use a fully implicit (backward Euler) scheme for both equations. This choice has several advantages. (1) It is physically sensible on fluid-flow time scales, because when $c \Delta t \gg \Delta r$, the radiation field is essentially quasistatic at the advanced time level. Indeed, experience shows (M8) that the fully implicit scheme should be used even on radiation-flow time scales because time-centered differencing tends to produce large, unphysical oscillations of the solution. (2) It maximizes stability; a von Neumann local stability analysis shows that the fully implicit equations are unconditionally stable. (3) It is algebraically simple; other choices lead to more complex equations (M8), (M10).

The fully implicit representation of the radiation momentum equation (98.8) is

$$
\begin{gathered}
F_{i}^{n+1}=-\beta_{i, i+(1 / 2)}^{n+1} E_{i+(1 / 2)}^{n+1}+\beta_{i, i-(1 / 2)}^{n+1} E_{i-(1 / 2)}^{n+1}+\alpha_{i}^{n+1} F_{i}^{n,} \\
(i=2, \ldots, I),
\end{gathered}
$$

where, writing $\gamma \equiv 1 / c \Delta t$,

and

$$
\alpha_{i}^{n+1} \equiv \gamma /\left(\gamma+\chi_{i}^{n+1}\right)
$$

$$
\beta_{i, i \pm(1 / 2)}^{n+1} \equiv 4 \pi c\left(r_{i}^{n+1}\right)^{2} \rho_{i}^{n+1} f_{i \pm(1 / 2)}^{n+1} q_{i \pm(1 / 2)}^{n+1} / q_{i}^{n+1}\left(\gamma+\chi_{i}^{n+1}\right) \Delta M_{i} .
$$

Here $\rho_{i}^{n+1}$ and $\chi_{i}^{n+1}$ are suitable averages across the interface $r_{i}$. For $\rho_{i}$ it is reasonable to adopt the mass-weighted average

$$
\rho_{i} \equiv\left[\rho_{i-(1 / 2)} \Delta M_{i-(1 / 2)}+\rho_{i+(1 / 2)} \Delta M_{i+(1 / 2)}\right] /\left[\Delta M_{i-(1 / 2)}+\Delta M_{i+(1 / 2)}\right] .
$$

Similarly, to obtain the correct optical-depth increment between cell centers one might adopt

$$
(\chi / \rho)_{i} \equiv\left[(\chi / \rho)_{i-(1 / 2)} \Delta M_{i-(1 / 2)}+(\chi / \rho)_{i-(1 / 2)} \Delta M_{i+(1 / 2)}\right] /\left[\Delta M_{i-(1 / 2)}+\Delta M_{i+(1 / 2)}\right] .
$$

For certain classes of problems, however, (98.26) is unsatisfactory. For example, in stellar pulsation calculations the temperature sensitivity of the opacity is so steep $\left(\chi \propto T^{12}\right)$ that there can be enormous (one or two orders of magnitude) jumps in the opacity between successive zones. In this case, 
Christy has shown (C4) [see also (S5)] that reasonably accurate flux transport is obtained in the diffusion limit by using the energy-weighted harmonic average

$$
(\rho / \chi)_{i} \equiv \frac{\left\{\left[T_{i-(1 / 2)}\right]^{4}(\rho / \chi)_{i-(1 / 2)}+\left[T_{i+(1 / 2)}\right]^{4}(\rho / \chi)_{i+(1 / 2)}\right\}}{\left\{\left[T_{i-(1 / 2)}\right]^{4}+\left[T_{i+(1 / 2)}\right]^{4}\right\}}
$$

For an inner boundary condition we fix the incident flux. If $r_{1}$ is the center of a star, $F_{1} \equiv 0$. If $r_{1}$ is the radius of a static core inside a dynamical envelope, we set $F_{1}=L_{1} / 4 \pi r_{1}^{2}$ where $L_{1}$ is assumed given. To obtain an outer boundary condition we apply (98.22) on the half-shell from $r_{1 \rightarrow(1 / 2)}$ to $R_{I+1}$; then

$$
F_{I-1}^{n+1}=-\tilde{\beta}_{I+1,1+1}^{n+1} E_{I+1}^{n+1}+\tilde{\beta}_{I+1, I+(1 / 2)}^{n+1} E_{I+(1 / 2)}^{n+1}+\tilde{\alpha}_{I+1}^{n+1} F_{I+1}^{n},
$$

where $\tilde{\alpha}$ and $\tilde{\beta}$ are defined by (98.23) and (98.24) with $\Delta M_{i}$ replaced by $\frac{1}{2} \Delta M_{\mathrm{T}+(1 / 2)}$. Invoking a geometrical closure of the form

$$
F_{I+1}^{n+1}=h_{I+1}^{n+1} c E_{I+1}^{n+1}
$$

where $h$ is given by the formal solution, as in (83.45), we find

where

$$
F_{I+1}^{n+1}=\beta_{I+1, I+(1 / 2)}^{n+1} E_{I+(1 / 2)}^{n+1}+\alpha_{I+1}^{n+1} F_{I+1}^{n},
$$

$$
\alpha_{I+1}^{n+1} \equiv c h_{I+1}^{n+1} \tilde{\alpha}_{I+1}^{n+1} /\left(c h_{I+1}^{n+1}+\tilde{\beta}_{I+1, I+1}^{n+1}\right)
$$

and

$$
\beta_{I+1, I \pm(1 / 2)}^{n+1} \equiv c h_{I+1}^{n+1} \tilde{\beta}_{I+1, I \pm(1 / 2)}^{n+1} /\left(c h_{I+1}^{n+1}+\tilde{\beta}_{I+1, I+1}^{n+1}\right) .
$$

The fully implicit representation of the radiation energy equation (98.8) is

$$
\begin{aligned}
{[} & \left.E_{i+(1 / 2)}^{n+1} / \rho_{i+(1 / 2)}^{n+1}\right]+\llbracket f_{i+(1 / 2)}^{n+1}\left\{\left[1 / \rho_{i+(1 / 2)}^{n+1}\right]-\left[1 / \rho_{i-(1 / 2)}^{n}\right]\right\} \\
& \left.-\left[3 f_{i+(1 / 2)}^{n+1}-1\right](v / r)_{i+(1 / 2)}^{n+(1 / 2)} \Delta t^{n+(1 / 2)} / \rho_{i+(1 / 2)}^{n+1}\right] E_{i+(1 / 2)}^{n+1} \\
= & {\left[E_{i+(1 / 2)}^{n} / \rho_{i+(1 / 2)}^{n}\right]+\Delta t^{n+(1 / 2)}\left\{\left[4 \pi \kappa_{P, i+(1 / 2)}^{n+1} B_{i+(1 / 2)}^{n+1}-c \kappa_{E, i+(1 / 2)}^{n+1} E_{i+(1 / 2)}^{n+1}\right] / \rho_{i+(1 / 2)}^{n+1}\right.} \\
& \left.-4 \pi\left[\left(r_{i+1}^{n+1}\right)^{2} F_{i+1}^{n+1}-\left(r_{i}^{n+1}\right)^{2} F_{i}^{n+1}\right] / \Delta M_{i+(1 / 2)}\right\}, \quad(i=1, \ldots, I) .
\end{aligned}
$$

Equations (98.22), (98.30), and (98.33) are a coupled system for $F_{i}^{n+1}$, $(i=1, \ldots, I+1)$, and $E_{i+(1 / 2)}^{n+1},(i=1, \ldots, I)$. Alternatively we can use (98.22) and (98.30) in (98.33) to eliminate the $F_{i}$ 's analytically, obtaining a finite difference representation of the combined moment equation (98.12). Thus for interior shells $(i=2, \ldots, I-1)$ we have

$$
\begin{aligned}
{[} & \left.\left.E_{i+(1 / 2)}^{n+1} / \rho_{i+(1 / 2)}^{n+1}\right]+\left[f_{i+(1 / 2)}^{n+1}\left\{\left[1 / \rho_{i+1}^{n+1}\right]-[1 / 2)\right] \rho_{i+(1 / 2)}^{n}\right]\right\} \\
& \left.-\left[3 f_{i+(1 / 2)}^{n+1}-1\right](v / r)_{i+(1 / 2)}^{n+(1 / 2)} \Delta t^{n+(1 / 2)} / \rho_{i+(1 / 2)}^{n+1}\right] E_{i+(1 / 2)}^{n+1} \\
= & {\left[E_{i+(1 / 2)}^{n+} / \rho_{i+(1 / 2)}^{n}\right]+\Delta t^{n+(1 / 2)}\left[\left[4 \pi \kappa_{P, i+(1 / 2)}^{n+1} B_{i+(1 / 2)}^{n+1}-c \kappa_{E, i+(1 / 2)}^{n+1} E_{i+(1 / 2)}^{n+1}\right] / \rho_{i+(1 / 2)}^{n+1}\right.} \\
& +4 \pi\left\{\left(r_{i-1}^{n+1}\right)^{2}\left[\beta_{i+1, i+(3 / 2)}^{n+1} E_{i+(3 / 2)}^{n+1}-\beta_{i+1, i+(1 / 2)}^{n+1} E_{i+(1 / 2)}^{n+1}-\alpha_{i+1}^{n+1} F_{i+1}^{n}\right]\right. \\
& \left.\left.-\left(r_{i}^{n+1}\right)^{2}\left[\beta_{i, i+(1 / 2)}^{n+1} E_{i+(1 / 2)}^{n+1}-\beta_{i, i-(1 / 2)}^{n+1} E_{i+(1 / 2)}^{n+1}-\alpha_{i}^{n+1} F_{i}^{n}\right]\right\} / \Delta M_{i+(1 / 2)}\right] .
\end{aligned}
$$


For the innermost shell $(i=1)$ we have

$$
\begin{aligned}
& \left(E_{3 / 2}^{n+1} / \rho_{3 / 2}^{n+1}\right)+\left\{f_{3 / 2}^{n+1}\left[\left(1 / \rho_{3 / 2}^{n+1}\right)-\left(1 / \rho_{3 / 2}^{n}\right)\right]\right. \\
& \left.\quad-\left(3 f_{3 / 2}^{n+1}-1\right)(v / r)_{3 / 2}^{n+(1 / 2)} \Delta t^{n+(1 / 2)} / \rho_{3 / 2}^{n+1}\right\} E_{3 / 2}^{n+1} \\
& =\left(E_{3 / 2}^{n} / \rho_{3 / 2}^{n}\right)+\Delta t^{n+(1 / 2)}\left\{\left(4 \pi \kappa_{P, 3 / 2}^{n+1} B_{3 / 2}^{n+1}-c \kappa_{E, 3 / 2}^{n+1} E_{3 / 2}^{n+1}\right) / \rho_{3 / 2}^{n+1}\right. \\
& \left.\quad+\left[4 \pi\left(r_{2}^{n+1}\right)^{2}\left(\beta_{2,5 / 2}^{n+1} E_{5 / 2}^{n+1}-\beta_{2,3 / 2}^{n+1} E_{3 / 2}^{n+1}-\alpha_{2}^{n+1} F_{2}^{n}\right)+L_{1}\right] / \Delta M_{3 / 2}\right\},
\end{aligned}
$$

and for the outermost shell $(i=I)$,

$$
\begin{aligned}
& {\left[E_{I+(1 / 2)}^{n+1} / \rho_{I+(1 / 2)}^{n+1}\right]+\left[f_{I+(1 / 2)}^{n+1}\left\{\left[1 / \rho_{I+(1 / 2)}^{n+1}\right]-\left[1 / \rho_{I+(1 / 2)}^{n}\right]\right\}\right.} \\
&\left.-\left[3 f_{I+(1 / 2)}^{n+1}-1\right](v / r)_{I+(1 / 2)}^{n+(1 / 2)} \Delta t^{n+(1 / 2)} / \rho_{I+(1 / 2)}^{n+1}\right] E_{I+(1 / 2)}^{n+1} \\
&= {\left[E_{I+(1 / 2)}^{n+1} / \rho_{I+(1 / 2)}^{n}\right] } \\
&+\Delta t^{n+(1 / 2)} \llbracket\left[4 \pi \kappa_{P, i+(1 / 2)}^{n+1} B_{i+(1 / 2)}^{n+1}-c \kappa_{E I I+(1 / 2)}^{n+1} E_{i+(1 / 2)}^{n+1}\right] / \rho_{I+(1 / 2)}^{n+1} \\
&-4 \pi\left\{\left(r_{I+1}^{n+1}\right)^{2}\left[\beta_{I+1, I+(1 / 2)}^{n+1} E_{I+(1 / 2)}^{n+1}+\alpha_{I+1}^{n+1} F_{I+1}^{n}\right]\right. \\
&\left.\left.\left.+\left(r_{I}^{n}\right)^{2}\left[\beta_{I, I+(1 / 2)}^{n-1} E_{I+(1 / 2)}^{n+1}-\beta_{I, I-(1 / 2)}^{n+1} E_{I-(1 / 2)}^{n+1}-\alpha_{I}^{n+1} F_{I}^{n}\right]\right\} / \Delta M_{I+(1 / 2)}\right)\right]
\end{aligned}
$$

To evaluate the cell-centered quantity $(v / r)_{i+(1 / 2)}$ in (98.33) to (98.36) we define $r_{i \pm(1 / 2)}$ as in (83.86), whence by differentiation with respect to time we have

$$
(v / r)_{i+(1 / 2)}=\left(r_{i}^{2} v_{i}+r_{i+1}^{2} v_{i+1}\right) /\left(r_{i}^{3}+r_{i+1}^{3}\right) .
$$

For calculations on a nuclear-evolutionary or an acoustic time scale, $\gamma \Delta r \ll 1$ and $\gamma \lambda_{p} \ll 1$, hence we can usually set $\gamma=0$ in the radiation momentum equation. For phenomena on much shorter time scales (e.g., accretion onto compact objects), $\gamma \Delta r$ may approach unity, and $\gamma \lambda_{\mathrm{p}}$ may greatly exceed unity in transparent regions of the flow; the time derivative in the radiation momentum equation must then be kept.

Equations (98.22), (98.30), and (98.33), plus the material energy equation $(98.20$ ) can be assembled into a system of nonlinear equations for the unknowns $T_{i+(1 / 2)}^{n+1}, E_{i+(1 / 2)}^{n+1}$, and $F_{i+1}^{n+1},(i=1, \ldots, I)$. We linearize the system around trial estimates $\left[T_{i+(1 / 2)}^{*}, E_{i+(1 / 2)}^{*}, F_{i+1}^{*}\right]$ to obtain a block tridiagonal system of $(3 \times 3)$ matrices coupling a set of solution vectors $\delta \psi_{i} \equiv\left[\delta T_{i+(1 / 2)}, \delta E_{i+(1 / 2)}, \delta F_{i+1}\right],(i=1, \ldots, I)$; the linearized system is solved by Gaussian elimination and iterated to consistency. In this procedure we might write $\kappa_{E} \equiv k_{E} \kappa_{P}$ and $\chi_{F} \equiv k_{F} \chi_{R}$, and linearize $\kappa_{P}$ and $\chi_{R}$ as functions of $T$, assuming that the ratios $k_{E}$ and $k_{F}$ determined in the multigroup step remain fixed. Alternatively we might calculate $\left(\partial \kappa_{E} / \partial T\right)$ from $\left(\partial \kappa_{\mathrm{g}} / \partial T\right)$ in each group weighted by the spectral profile $\boldsymbol{e}_{\mathrm{g}}$, and similarly for $\left(\partial \chi_{F} / \partial T\right)$.

If the combined moment equation is used instead of the individual radiation energy and momentum equations, the unknowns are reduced to $(T, E)_{i+(1 / 2)}^{n+1},(i=1, \ldots, I)$. The $F_{i}^{n+1}$,s can be calculated from the $E_{i+(1 / 2)}^{n+1}$ 's using (98.22) and (98.30). The method of solution is the same, but the matrices are now only $(2 \times 2)$. The advantage of the smaller matrix size may, however, be offset by the more complicated structure of the linearized equations. 
If the hydrodynamic equations are coupled to the radiation equations in implicit, rather than explicit, form, we must determine simultaneously $r_{i}^{n+1}$, $v_{i}^{n+1}, \rho_{i+-(1 / 2)}^{n+1}, T_{i+(1 / 2)}^{n+1}, E_{i+(1 / 2)}^{n+1}$, and $F_{i+(1 / 2)}^{n+1}$. When linearized, this set of equations produces a block tridiagonal system of $(6 \times 6)$ matrices coupling solution vectors $\delta \psi_{i} \equiv\left[\delta r_{i-1}, \delta v_{i+1}, \delta \rho_{i+(1 / 2)}, \delta T_{i+(1 / 2)}, \delta E_{i+(1 / 2)}, \delta F_{i+1}\right], \quad(i=$ $1, \ldots, I)$. The higher cost of solving this larger system at each timestep may be offset by having to take many fewer timesteps because we no longer need to observe the Courant condition imposed on the explicit scheme.

ENERGY CONSERVATION

Equation (96.15) is an expression of total energy conservation for a radiating fluid. Integrating over mass we have

$$
\frac{D}{D t} \int\left(e+\frac{E}{\rho}+\frac{1}{2} v^{2}-\frac{G M_{r}}{r}\right) d M_{r}+4 \pi r_{I+1}^{2} v_{I+1} P_{I+1}+L_{I-1}-L_{1}=\int \varepsilon d M_{r} .
$$

Here, for simplicity, we have assumed that the inner boundary is fixed $\left(v_{1} \equiv 0\right)$, and that the gas and pseudoviscous pressure vanish at the outer boundary. Introducing discretized variables, and replacing integrals over mass and time by sums, we obtain the total energy conservation law

$$
\begin{aligned}
\mathscr{E}^{n+1}= & \sum_{i} \llbracket\left\{e_{i-(1 / 2)}^{n+1}+\left[E_{i+(1 / 2)}^{n+1} / \rho_{i+(1 / 2)}^{n+1}\right]\right\} \Delta M_{i+(1 / 2)}+\left[\frac{1}{2}\left(v_{i}^{n+1}\right)^{2}-G M_{i} / r_{i}^{n}\right] \Delta M_{i} \rrbracket \\
& +W_{1+1}^{n+1}+\mathscr{L}^{n+1}-q^{n+1}=\mathrm{constant},
\end{aligned}
$$

where

$$
\begin{gathered}
\mathcal{W}_{I+1}^{n+1} \equiv \sum_{k=0}^{n} \Delta t^{k+(1 / 2)}\left(\frac{4}{3} \pi\right)\left[\left(r_{I+1}^{k+1}\right)^{3}-\left(r_{I+1}^{k}\right)^{3}\right] \frac{1}{2}\left(P_{I+1}^{k}+P_{I+1}^{k+1}\right), \\
\mathscr{L}^{n+1} \equiv \sum_{k=0}^{n} \Delta t^{k+(1 / 2)}\left[\left(1-\theta^{\prime}\right)^{k+(1 / 2)}\left(L_{I+1}^{k}-L_{1}^{k}\right)+\theta^{\prime k+(1 / 2)}\left(L_{I+1}^{k+1}-L_{1}^{k+1}\right)\right]
\end{gathered}
$$

and

$$
q^{n+1} \equiv \sum_{k=0}^{n} \Delta t^{k+(1 / 2)} \sum_{i}\left[(1-\theta)^{k+(1 / 2)} \varepsilon_{i+(1 / 2)}^{k}+\theta^{k+(1 / 2)} \varepsilon_{i+(1 / 2)}^{k+1}\right] \Delta M_{i+(1 / 2)} .
$$

The difference equations do not explicitly guarantee (98.39). Instead, we evaluate $\mathscr{E}^{n+1}$ after each integration step, and use its constancy as a check on the quality of the solution.

FORMAL SOLUTION

The Eddington factors needed in the radiation energy and momentum equations are obtained from a formal solution of the angle-frequencydependent transfer equation using current estimates of source-sink terms. 
To obtain a solution accurate to $\mathrm{O}(v / \mathrm{c})$, we would need to solve (95.17) or (95.80), which would be difficult because these are partial differential equations in four independent variables, hence costly to solve [cf. (M4), (M12)]. We thus seek a simpler procedure.

The simplest approach is to drop both $(D / D t)$ and all velocity-dependent terms from the transfer equation. We then solve the static transfer problem along straight rays, as in $\$ 83$, using the instantaneous positions of mass shells and current values of all material properties. We thus obtain a snapshot of the radiation field, from which we can calculate $E_{\nu}$ and $P_{\nu}$, hence $f_{\nu}$ and $q_{\nu}$, at all depths and frequencies. For some problems it may even be possible to ignore curvature effects. For example, in a pulsating star the radiation field is anisotropic only in the atmosphere, which is usually so thin compared to a stellar radius that it can be assumed to be plane parallel; curvature effects become important deeper in the envelope (a significant fraction of the radius in), but here we can simply set $f_{\nu} \equiv \frac{1}{3}$.

Intuitively one expects static snapshots to be adequate for most astrophysical applications because the ratio $f_{v}$, should vary less rapidly than the individual values of $E_{v}$ and $P_{v}$. On the other hand, this approach is likely to be inadequate for flows that contain radiation fronts, that evolve on time scales of the same order as $t_{R}$, or that are transparent over such enormous distances that retardation effects are important (e.g., the cosmic expansion). Here we must at least retain information about the time variation of the radiation field.

For the purpose of computing Eddington factors we therefore propose a model Lagrangean transfer equation that (1) accounts for time dependence, and (2) is consistent with the radiation energy and momentum equations, but (3) omits inessential complications arising from ray curvature and Doppler shifts. We obtain such an equation by rewriting (95.80) as

$$
\begin{aligned}
& \frac{\rho}{c} \frac{D}{D t}\left(\frac{I_{\nu}}{\rho}\right)+4 \pi r^{2} \rho \mu \frac{\partial I_{v}}{\partial M_{r}}+\frac{\left(1-\mu^{2}\right)}{r} \frac{\partial I_{v}}{\partial \mu} \\
& \quad+\frac{1}{c}\left\{\left(\frac{3 v}{r}+\frac{D \ln \rho}{D t}\right) \frac{\partial}{\partial \mu}\left[\mu\left(1-\mu^{2}\right) I_{v}\right]-\left[\left(1-3 \mu^{2}\right) \frac{v}{r}-\mu^{2} \frac{D \ln \rho}{D t}\right] \frac{\partial\left(\nu I_{v}\right)}{\partial \nu}\right\} \\
& =\eta_{\nu}-\left[\chi_{\nu}+\left(1-3 \mu^{2}\right) \frac{v}{c r}-\frac{\mu^{2}}{c} \frac{D \ln \rho}{D t}\right] I_{\nu},
\end{aligned}
$$

and then dropping all of the terms in the braces which (1) are only $\mathrm{O}(v / \mathrm{c})$, and (2) vanish identically when integrated over angle and frequency. The remaining terms describe time-dependent transfer along straight rays, and include two velocity-dependent terms that account for the rate of work done by radiation pressure.

Forming symmetric and antisymmetric averages of (98.43) for $\pm \mu$ we 
have

$$
\frac{\rho}{c} \frac{D}{D t}\left(\frac{i_{v}}{\rho}\right)+4 \pi r^{2} \rho \mu \frac{\partial h_{v}}{\partial M_{r}}+\frac{\left(1-\mu^{2}\right)}{r} \frac{\partial h_{v}}{\partial \mu}=\eta_{\nu}-\left[\chi_{\nu}+\left(1-3 \mu^{2}\right) \frac{v}{c r}-\frac{\mu^{2}}{c} \frac{D \ln \rho}{D t}\right] j_{\nu},
$$

and

$$
\frac{\rho}{c} \frac{D}{D t}\left(\frac{h_{\nu}}{\rho}\right)+4 \pi r^{2} \rho \mu \frac{\partial j_{\nu}}{\partial M_{r}}+\frac{\left(1-\mu^{2}\right)}{r} \frac{\partial j_{\nu}}{\partial \mu}=-\left[\chi_{\nu}+\left(1-3 \mu^{2}\right) \frac{v}{c r}-\frac{\mu^{2}}{c} \frac{D \ln \rho}{D t}\right] h_{\nu} .
$$

We drop the velocity-dependent terms in (98.45), which are never larger than $\mathrm{O}(v / c)$ (cf. \$95), and use

$$
\frac{1}{c} \frac{D h_{v}}{D t}+4 \pi r^{2} \rho \mu \frac{\partial j_{v}}{\partial M_{r}}+\frac{\left(1-\mu^{2}\right)}{r} \frac{\partial j_{v}}{\partial \mu}=-\chi_{v} h_{v}
$$

In (98.44) to (98.46) we regard $j$ and $h$ as functions of $\left(M_{r}, t ; \mu, \nu\right)$.

When (98.44) is integrated over angle and frequency we recover the Lagrangean radiation energy equation (98.5); similarly (98.46) yields the Lagrangean radiation momentum equation (98.8). These equations thus meet all the desiderata stated above.

One approach to solving these equations is to rewrite them along tangent rays through cell centers, as in $\$ 83$. Then

$$
\frac{\rho}{c} \frac{D}{D t}\left(\frac{j_{\nu}}{\rho}\right)+\frac{\partial h_{v}}{\partial s}=\eta_{v}-\left[\chi_{v}+\left(1-3 \mu^{2}\right) \frac{v}{c r}-\frac{\mu^{2}}{c} \frac{D \ln \rho}{D t}\right] j_{v}
$$

and

$$
\frac{1}{c} \frac{D h_{\nu}}{D t}+\frac{\partial j_{\nu}}{\partial s}=-\chi_{\nu} h_{\nu}
$$

The computational effort to solve (98.47) and (98.48) for $I$ shells and $G$ groups scales as $C I^{2} G$. A complication with this method is that the angles $\left\{\mu_{\mathrm{r}}\right\}$ at which the tangent rays intersect mass shells change over $\Delta t^{n+(1 / 2)}$ because the shells move. To form time differences we need $j^{n}\left[M_{i+(1 / 2),} \mu_{i+(1 / 2), m}^{n+1}, \nu_{\mathrm{g}}\right]$, not $i^{n}\left[M_{i+(1 / 2),}, \mu_{i+(1 / 2), m}^{n}, \nu_{\mathrm{g}}\right]$. In principle one should interpolate $j$ and $h$ as functions of angle at $t^{n}$. However if the motion of the shells is small over $\Delta t^{12+(1 / 2)},(v / c \ll 1)$, the interpolation can be ignored, the resulting error being of the same order as made by neglecting the $\mathrm{O}(v / c)$ terms dropped in (98.44) and (98.46). The problem does not arise in planar geometry, where motions of the mass zones do not affect the direction of rays when aberration is neglected.

The alternative is to rewrite (98.44) and (98.46) in conservative form,

$$
\begin{aligned}
\frac{\rho}{c} \frac{D}{D t}\left(\frac{j_{\nu}}{\rho}\right)+4 \pi \rho \mu \frac{\partial}{\partial M_{r}}\left(r^{2} h_{\nu}\right) & +\frac{1}{r} \frac{\partial}{\partial \mu}\left[\left(1-\mu^{2}\right) h_{\nu}\right] \\
& =\eta_{\nu}-\left[\chi_{v}+\left(1-3 \mu^{2}\right) \frac{v}{c r}-\frac{\mu^{2}}{c} \frac{D \ln \rho}{D t}\right] j_{\nu}
\end{aligned}
$$


and

$$
\frac{\mu}{c} \frac{D h_{\nu}}{D t}+4 \pi \rho \mu^{2} \frac{\partial}{\partial M_{r}}\left(r^{2} j_{\nu}\right)+\frac{\left(\mu^{2}-1\right)}{r} j_{\nu}+\frac{1}{r} \frac{\partial}{\partial \mu}\left[\mu\left(\mu^{2}-1\right) j_{\nu}\right]=-\chi_{\nu} \mu h_{\nu}
$$

and then use a discrete representation on a radial mesh $\left\{r_{i}\right\}$, angle mesh $\left\{\mu_{m}\right\}$, and frequency mesh $\left\{\nu_{g}\right\}$, as in $\$ 83$. The computational effort required to solve these equations for $I$ shells, $M$ angles, and $G$ groups scales as $C I M^{3} G$.

Before considering the multigroup step it is worth noting that the comoving-frame transfer problem for spectral lines is rather different. Here Doppler shifts dominate all other $O(v / c)$ terms because the radiation field in a line changes markedly over a Doppler width $\Delta \nu_{D}$. Dimensional analysis shows that the $(\partial / \partial \nu)$ term is effectively amplified to $\mathrm{O}\left(v / v_{\mathrm{th}}\right)$ where $v_{\mathrm{th}}$ is of the order of the sound speed in the material; hence aberration and advection terms can be ignored. The comoving-frame line-transfer equation is then a partial differential equation in $(s, t, \nu)$ along straight rays, and is relatively easily solved for steady expansion (M9), (M11), (M13); however, for nonmonotonic flows the problem is much more complex (N1), and it may be preferable to work in the inertial frame (M5). Unfortunately, present prospects for a consistent treatment of the effects of line blanketing on energy and momentum balance in dynamical media are dim, despite their probable importance.

THE MULTIGROUP EQUATIONS

The formal solution yields estimates of $E_{\mathrm{g}}$ and $F_{\mathrm{g}}$ for all groups as a by-product. However these results may not yield satisfactory spectral profiles $e_{\mathrm{g}}$ and $f_{\mathrm{g}}$ because frequency derivatives were ignored in the formal solution. Instead, we should calculate $E_{\mathrm{g}}$ and $F_{\mathrm{g}}$ from the monochromatic moment equations including the frequency derivatives.

Because we do not require energy densities and fluxes simultaneously in this step it is computationally more efficient to work with the combined moment equation. By the same steps leading to (98.22) and (98.30) we can write (98.4) as

and

$$
\begin{gathered}
F_{i \mathrm{~g}}^{n+1}=-\beta_{i, i+(1 / 2), \mathrm{g}}^{n+1} E_{i+(1 / 2), \mathrm{g}}^{n+1}+\beta_{i, i-(1,2), \mathrm{g}}^{n+1} E_{i-(1 / 2), \mathrm{g}}^{n+1}+\alpha_{i \mathrm{~g}}^{n+1} F_{i \mathrm{~g}}^{n}, \\
(i=2, \ldots, I),
\end{gathered}
$$

$$
F_{I+i, g}^{n+1}=\beta_{I+1, I+(1 / 2), g}^{n+1} E_{I+(1 / 2), g}^{n+1}+\alpha_{I-1, g}^{n+1} F_{I+1, \mathrm{~g}}^{n}
$$

where the $\alpha$ 's and $\beta$ 's are defined in (98.23), (98.24), (98.31), and (98.32), except that frequency (group) subscripts are appended to $f, q$, and $\chi$. At the inner boundary we can apply the planar diffusion because $\lambda_{\mathrm{p}} \ll r_{\mathrm{1}}$.

$$
F_{\mathrm{lg}}^{n+1}=\left(\frac{\chi_{\mathrm{R}}^{n+1}}{\bar{\chi}_{\mathrm{g}}^{n+1}}\right)_{1} \frac{\left(\partial B_{\nu} / \partial T\right)_{\mathrm{lg}}^{n+1}}{(d B / d T)_{1}^{n+1}}\left(\frac{L_{1}}{4 \pi r_{1}^{2}}\right)
$$


Using (98.51) to (98.53) to eliminate fluxes from the discrete representation of (98.1) we obtain the multigroup combined moment equation

$$
\begin{aligned}
& {\left[E_{i+(1 / 2), g}^{n+1} / \rho_{i+(1 / 2)}^{n+1}\right]+\llbracket f_{i+(1 / 2), g}^{n+1}\left\{\left[1 / \rho_{i+(1 / 2)}^{n+1}\right]-\left[1 / \rho_{i+(1 / 2)}^{n}\right]\right\}} \\
& -\left[3 f_{i+(1 / 2), g}^{n+1}-1\right](v / r)_{i+(1 / 2)}^{n+(1 / 2)} \Delta t^{n+(1 / 2)} / \rho_{i+(1 / 2)}^{n+1} \rrbracket E_{i+(1 / 2), g}^{n+1}=\left[E_{i+(1 / 2), g}^{n} / \rho_{i+(1 / 2)}^{n}\right] \\
& +\Delta t^{n+(1 / 2)} \mathbb{U}\left[4 \pi \kappa_{P, i+(1 / 2), g}^{n+1} B_{i+(1 / 2), g}^{n+1}-c \bar{\kappa}_{i+(1 / 2), g}^{n+1} E_{i+(1 / 2), g}^{n-1}\right] / \rho_{i+(1 / 2)}^{n+1} \\
& +4 \pi\left\{\left(r_{i+1}^{n+1}\right)^{2}\left[\beta_{i+1, i+(3 / 2), g}^{n+1} E_{i+(3 / 2), g}^{n+1}-\beta_{i+1, i+(1 / 2), g}^{n+1} E_{i+(1 / 2), g}^{n+1}-\alpha_{i+1, g}^{n+1} F_{i+1, g}^{n}\right]\right. \\
& \left.-\left(r_{i}^{n+1}\right)^{2}\left[\beta_{i, i+(1 / 2), \mathrm{g}}^{n+1} E_{i+(1 / 2), \mathrm{g}}^{n+1}-\beta_{i, i-(1 / 2), \mathrm{g}}^{n+1} E_{i-(1 / 2), \mathrm{g}}^{n+1}-\alpha_{i g}^{n+1} F_{i g}^{n}\right]\right\} / \Delta M_{i+(1 / 2)} \Pi \\
& +\left\{\left[1 / \rho_{i+(1 / 2)}^{n+1}\right]-\left[1 / \rho_{i+(1 / 2)}^{n}\right]\right\} \Delta_{\mathrm{g}}\left(v f_{\nu} E_{\nu}\right)_{i+(1 / 2)}^{n+1} \\
& -(v / r)_{i+(1 / 2)}^{n+(1 / 2)} \Delta t^{n+(1 / 2)} \Delta_{\mathrm{g}}\left[\nu\left(3 f_{\nu}-1\right) E_{\nu}\right]_{i+(1 / 2)}^{n+1} / \rho_{i+(1 / 2)}^{n+1}, \\
& (i=2, \ldots, I-1 ; g=1, \ldots, G) \text {. }
\end{aligned}
$$

The $\Delta_{8}$ 's in (98.54) are to be written using upstream differencing, as in (97.86). Analysis of (98.1) shows that the effective "advection velocity" of photons in frequency space is

$$
(D \nu / D t)=\left[f_{\nu}(D \ln \rho / D t)+\left(3 f_{v}-1\right)(v / r)\right] \nu .
$$

If $(D \nu / D t)>0$, photons are shifted to higher frequencies during the timestep, and writing $x_{v}$ for either $f_{\nu}$ or $\left(3 f_{\nu}-1\right)$ we choose

$$
\Delta_{\mathrm{g}}\left(\nu x_{\nu} E_{v}\right)=\left[\nu_{\mathrm{g}+(1 / 2)} / \Delta \nu_{\mathrm{g}}\right] x_{\mathrm{g}} E_{\mathrm{g}}-\left[\nu_{\mathrm{g}-(1 / 2)} / \Delta \nu_{\mathrm{g}}\right] x_{\mathrm{g}-1} E_{\mathrm{g}-1}
$$

if $(D \nu / D t)<0$ we choose

$$
\Delta_{\mathrm{g}}\left(\nu x_{v} E_{\nu}\right)=\left[\nu_{\mathrm{g}+(3 / 2)} / \Delta \nu_{\mathrm{g}+1}\right] x_{\mathrm{g}+1} E_{\mathrm{g}+1}-\left[\nu_{\mathrm{g}+(1 / 2)} / \Delta \nu_{\mathrm{g}}\right] x_{\mathrm{g}} E_{\mathrm{g}}
$$

Equation (98.55) shows that the sign of the frequency shift is not necessarily the same as $(D \rho / D t)$, as it is in the diffusion limit, and may even be different for different frequencies at a given position in the flow. This property implies that the difference equations (98.54) may not be exactly conservative when summed over frequency.

Boundary conditions are obtained by applying the discrete representation of (98.1) at $i=1$ and $i=I$, using (98.52) and (98.53) to eliminate $F_{\mathrm{lg}}^{n+1}$ and $F_{I+1 . g}^{n+1}$.

To handle the frequency coupling economically, we put the $\Delta_{\mathrm{g}}$ terms on the right-hand side along with known source terms, and solve the equations iteratively. As a first estimate we set $E_{\mathrm{g}}$ in $\Delta_{\mathrm{g}}$ (only) to $E_{\mathrm{g}}^{n+1} \approx e_{\mathrm{g}}^{n} E^{n+1}$, and solve the resulting $G$ systems in parallel. We use the new $E_{\mathrm{g}}$ 's to reevaluate the $\Delta_{\mathrm{g}}$ 's, and iterate the solution to convergence. For the continuum this procedure converges quickly because the frequencyderivative terms result only in a minor redistribution of energy among bins. For spectral lines (e.g., included in an ODF) this is not the case, but we cannot pursue this point further here.

If $J$ iterations are required to achieve convergence, the computational 
effort in this step scales as $c J I G$. If one performs a direct solution to handle the coupling between groups instead of the iterative procedure just described, the computational effort would scale as $c I G^{3}$, which is probably prohibitively expensive for dynamical calculations (as was also true for multigroup diffusion).

Having found $E_{i+(1 / 2), g}^{n+1}$, we calculate $F_{i g}^{n-1}$ for all $i$ and $g$ from (98.51) and (98.52). We can then update the spectral profiles $e_{\mathrm{g}}$ and $f_{\mathrm{g}}$, and use these to re-evaluate $\chi_{F}$ and $\kappa_{E}$. Finally, given the radiation field and source-sink terms in the comoving fluid frame, one must remember to transform back to the inertial frame when calculating the specific intensity or flux seen by an external observer; this transformation is particularly important in spectral lines.

\section{Transport Solution by Mixed-Frame and VERA-Code Methods}

In the mixed-frame method (cf. \$93) the specific intensity, angles, and frequencies are measured in the lab frame, while material properties are evaluated in the fluid frame by an expansion procedure. All velocitydependent terms then appear only on the right-hand side of the equations. The method is fundamentally Eulerian, though it is possible to cast it into a quasi-Lagrangean form.

In the VERA (Variable Eddington Radiation Approximation) method, which was developed before the Lagrangean equations of $\$ 95$ had been derived, the approach was to use a lab-frame spacetime operator, and fixed lab-frame angles and frequencies, while evaluating both the radiation field and material properties in the comoving frame. The method thus entails expansions of both radiation and material quantities, and the resulting equations are complicated, containing velocity-dependent terms both on the right-hand side and inside the differential operator. The physical meaning of the equations is often obscure, and in retrospect a pure Lagrangean formulation is clearly preferable. Nevertheless the method merits discussion because for many years VERA was the only code that handled $\mathrm{O}(v / c)$ terms, and was the source of many innovative techniques such as variable Eddington factors and the multifrequency/grey method.

THE MIXED-FRAME METHOD

The mixed-frame transfer equation for one-dimensional spherically symmetric flow is [cf. (93.4)]

$$
c^{-1}\left(\partial I_{v} / \partial t\right)+\mu\left(\partial I_{v} / \partial r\right)+r^{-1}\left(1-\mu^{2}\right)\left(\partial I_{v} / \partial \mu\right)=\eta_{v}-\kappa_{v} I_{v}+(\mu v / c)\left(\tilde{\kappa}_{v} I_{v}+\tilde{\eta}_{v}\right)
$$

where

and

$$
\tilde{\kappa}_{\nu} \equiv \kappa_{\nu}+\nu\left(\partial \kappa_{\nu} / \partial \nu\right)
$$

$$
\tilde{\eta}_{v} \equiv 3 \eta_{\nu}-\left[\partial\left(\nu \eta_{\nu}\right) / \partial \nu\right]
$$


Here we have omitted the affix " 0 " on material quantities, with the understanding that all material properties are evaluated in the comoving frame; furthermore we have ignored scattering $\left(\sigma_{\nu} \equiv 0\right)$ so that $\chi_{\nu} \equiv \kappa_{\nu}$.

For simplicity we assume that the opacity is represented by an opacity distribution function so that $\kappa_{v} \equiv \kappa_{\mathrm{g}}$ on $\left(\nu_{\mathrm{g}}, \nu_{\mathrm{g}+1}\right)$. Then the multigroup version of (99.1) is

$$
c^{-1}\left(\partial I_{\mathrm{g}} / \partial t\right)+\mu\left(\partial I_{\mathrm{g}} / \partial r\right)+r^{-1}\left(1-\mu^{2}\right)\left(\partial I_{\mathrm{g}} / \partial \mu\right)=\eta_{\mathrm{g}}-\kappa_{\mathrm{g}} I_{\mathrm{g}}+(\mu v / c)\left(\kappa_{\mathrm{g}} I_{\mathrm{g}}+\tilde{\eta}_{\mathrm{g}}\right),
$$

where $\eta_{\mathrm{g}}=\kappa_{\mathrm{g}} B_{\mathrm{g}}, B_{\mathrm{g}}$ is given by $(82.39)$, and

$$
\tilde{\eta}_{\mathrm{g}} \equiv \int_{\nu_{\mathrm{g}}}^{\nu_{\mathrm{g}+1}} \tilde{\eta}_{\nu} d \nu=3 \kappa_{\mathrm{g}} B_{\mathrm{g}}+\left[\nu_{\mathrm{g}+1} \eta\left(\nu_{\mathrm{g}+1}\right)-\nu_{\mathrm{g}} \eta\left(\nu_{\mathrm{g}}\right)\right] \equiv 3 \kappa_{\mathrm{g}} \tilde{B}_{\mathrm{g}}
$$

Equation (99.3) is written in conservative form so that the term in square brackets vanishes identically when integrated over $(0, \infty)$. To assure this property in the multigroup equations one can either (1) drop the term in square brackets in (99.5), which will be small if the frequency spectrum of the opacity is smooth and $\left(\nu_{\mathrm{g}+1}-\nu_{\mathrm{g}}\right)$ is not too large, or (2) choose an appropriate definition of $\eta\left(\nu_{\mathrm{g}}\right)$, for example, $\eta\left(\nu_{\mathrm{g}}\right) \equiv \frac{1}{2}\left(\kappa_{\mathrm{g}-1} B_{\mathrm{g}-1}+\kappa_{\mathrm{g}} B_{\mathrm{g}}\right)$.

Taking symmetric and antisymmetric averages of (99.4) for $\pm \mu$ we find

$$
c^{-1}\left(\partial j_{\mathrm{g}} / \partial t\right)+\mu\left(\partial h_{\mathrm{g}} / \partial r\right)+r^{-1}\left(1-\mu^{2}\right)\left(\partial h_{\mathrm{g}} / \partial \mu\right)=\kappa_{\mathrm{g}}\left(B_{\mathrm{g}}-j_{\mathrm{g}}\right)+(\mu v / c) \kappa_{\mathrm{g}} h_{\mathrm{g}},
$$

and

$$
c^{-1}\left(\partial h_{\mathrm{g}} / \partial t\right)+\mu\left(\partial j_{\mathrm{g}} / \partial r\right)+r^{-1}\left(1-\mu^{2}\right)\left(\partial j_{\mathrm{g}} / \partial \mu\right)=-\kappa_{\mathrm{g}} h_{\mathrm{g}}+(\mu v / c) \kappa_{\mathrm{g}}\left(j_{\mathrm{g}}+3 \tilde{B}_{\mathrm{g}}\right) .
$$

Integrating these equations over angle we obtain the multigroup energy equation

$$
\left(\partial E_{\mathrm{g}} / \partial t\right)=-\left(1 / r^{2}\right)\left[\partial\left(r^{2} F_{\mathrm{g}}\right) / \partial r\right]+\kappa_{\mathrm{g}}\left(4 \pi B_{\mathrm{g}}-c E_{\mathrm{g}}\right)+(v / c) \kappa_{\mathrm{g}} F_{\mathrm{g}} \equiv R_{\mathrm{g}},
$$

and momentum equation

$$
c^{-2}\left(\partial F_{\mathrm{g}} / \partial t\right)+\left(1 / q_{\mathrm{g}}\right)\left[\partial\left(f_{\mathrm{g}} q_{\mathrm{g}} E_{\mathrm{g}}\right) / \partial r\right]=-\left(\kappa_{\mathrm{g}} / c\right) F_{\mathrm{g}}+(v / c) \kappa_{\mathrm{g}}\left[f_{\mathrm{g}} E_{\mathrm{g}}+(4 \pi / c) \tilde{B}_{\mathrm{g}}\right],
$$

where $f_{\mathrm{g}} \equiv P_{\mathrm{g}} / E_{\mathrm{g}}$, and $q_{\mathrm{g}}$ is defined as in (98.3).

Integrating (99.8) and (99.9) over frequency we obtain the radiation energy equation

$$
(\partial E / \partial t)=-\left(1 / r^{2}\right)\left[\partial\left(r^{2} F\right) / \partial r\right]+4 \pi \kappa_{p} B-c \kappa_{E} E+(v / c) \kappa_{F} F \equiv R
$$

and the radiation momentum equation

$$
c^{-2}(\partial F / \partial t)+(1 / q)[\partial(f q E) / \partial r]=-\left(\kappa_{F} / c\right) F+(v / c)\left[\kappa_{K} f E+(4 \pi / c) \kappa_{P} B\right]
$$


where $f$ is defined by $(98.6), q$ by $(98.9), \kappa_{E}$ by $(98.7), \kappa_{F}$ by $(98.10)$, and

$$
\kappa_{K} \equiv\left(\int \kappa_{\nu} K_{\nu} d \nu / K\right)=\left(\sum_{\mathrm{B}} e_{\mathrm{g}} f_{\mathrm{g}} \kappa_{\mathrm{g}}\right) / f
$$

All terms in (99.10) and (99.11) must be kept in order to account for the work done by radiation forces, and to get the correct flux.

These radiation equations can be coupled to the Eulerian equation of continuity (19.9), momentum equation [cf. (94.14)]

$$
\frac{\partial(\rho v)}{\partial t}+\frac{\partial}{\partial r}\left(\rho v^{2}+p\right)+\frac{1}{q} \frac{\partial(f q E)}{\partial r}=-\frac{G M_{r} \rho}{r^{2}},
$$

and total energy equation [cf. (94.16)]

$$
\frac{\partial}{\partial t}\left(\rho e+\frac{1}{2} \rho v^{2}+E\right)+\frac{1}{r^{2}} \frac{\partial}{\partial r}\left[r^{2}\left(\rho e+\frac{1}{2} \rho v^{2}+p\right) v+r^{2} F\right]=\frac{-G M_{r} \rho v}{r^{2}},
$$

The computational strategy is essentially the same as for the Lagrangean scheme $(\$ 98)$ or for multigroup diffusion $(\$ 97)$. We solve the coupled nonlinear hydrodynamic and radiation energy and momentum equations, assuming that Eddington factors and spectral distributions are known. We then update the Eddington factors in a formal solution and obtain new spectral distributions from the multigroup moment equations. The process is iterated to convergence.

A wide variety of methods can be used to solve the Eulerian hydrodynamic equations, including special techniques to guarantee conservation and to handle shocks. These are discussed thoroughly in (R1, Chap. 12) and (R2, Chap. 5). In the absence of radiation one can use effectively explicit techniques like the Lax-Wendroff method; with radiation it is better to use implicit schemes such as outlined in (K4) [see also (T3)]. The momentum and total energy equations given in $(\mathbf{K 4})$ are easily generalized to include all the radiation terms written in (99.13) and (99.14), but these authors assume that the radiation field is quasi-static at the advanced time level, and ignore $\mathrm{O}(v / c)$ terms in the radiation equations. It is therefore worthwhile to write difference representations of the radiation equations here.

As before, we center fluxes on spherical shells $\left\{r_{i}\right\},(i=1, \ldots, I+1)$ and energy densities at cell centers $\left\{r_{i+(1 / 2)}\right\},(i=1, \ldots, I)$, defined by $(83.86)$. For the radiation energy equation we have

$$
\begin{aligned}
& {\left[E_{i+(1 / 2)}^{n+1}-E_{i+(1 / 2)}^{n}\right] / \Delta t^{n+(1 / 2)}=3\left[\left(r_{i+1}^{n+1}\right)^{2} F_{i+1}^{n+1}-\left(r_{i}^{n+1}\right)^{2} F_{i}^{n+1}\right] /\left[\left(r_{i+1}^{n+1}\right)^{3}-\left(r_{i}^{n+1}\right)^{3}\right] } \\
&+4 \pi \kappa_{P, i+(1 / 2)}^{n+1} B_{i+(1 / 2)}^{n-1}-c \kappa_{E, i+(1 / 2)}^{n+1} E_{i+(1 / 2)}^{n+1}(99.15) \\
&+ \frac{1}{2}\left(v_{i}^{n+1} \kappa_{F, i}^{n+1} F_{i}^{n+1}+v_{i+1}^{n+1} \kappa_{F, i+1}^{n+1} F_{i+1}^{n+1}\right) / c, \quad(i=1, \ldots, I),
\end{aligned}
$$


and for the radiation momentum equation we write

$$
\begin{aligned}
(\gamma+ & \left.\kappa_{F, i}^{n+1}\right) F_{i}^{n+1} \\
= & -c\left[f_{i+(1 / 2)}^{n-1} q_{i+(1 / 2)}^{n+1} E_{i+(1 / 2)}^{n+1}-f_{i-(1 / 2)}^{n+1} q_{i-(1 / 2)}^{n+1} E_{i-(1 / 2)}^{n+1}\right] /\left\{q_{i}\left[r_{i+(1 / 2)}^{n-1}-r_{i-(1 / 2)}^{n+1}\right]\right\} \\
& +v_{i}^{n+1}\left[\left\langle\kappa_{K} f E\right\rangle_{i}^{n+1}+(4 \pi / c)\left\langle\kappa_{p} B\right\rangle_{i}^{n+1}\right]+\gamma F_{i}^{n}, \quad(i=2, \ldots, I) \ldots(99.16)
\end{aligned}
$$

In (99.16), $x\rangle_{i} \equiv \frac{1}{2}\left[x_{i-(1 / 2)}+x_{i+(1 / 2)}\right]$, and $\gamma \equiv 1 /\left[c \Delta t^{n+(1 / 2)}\right]$.

Assembling the difference representations of the hydrodynamic and radiation equations we get a tridiagonal nonlinear system, which is linearized and solved for corrections $\left[\delta \rho_{i+(1 / 2)}, \delta v_{i+1}, \delta T_{i+(1 / 2)}, \delta E_{i+(1 / 2)}\right.$, $\left.\delta F_{i+1}\right],(i=1, \ldots, I)$ to current estimates of these quantities. Using the current estimate of the temperature distribution we calculate source-sink terms and carry out a formal solution at each frequency. As in the Lagrangean case we can either do a solution along tangent rays with a system of the form

and

$$
c^{-1}\left(\partial j_{\mathrm{g}} / \partial t\right)+\left(\partial h_{\mathrm{g}} / \partial s\right)=\kappa_{\mathrm{g}}\left[\left(B_{\mathrm{g}}-j_{\mathrm{g}}\right)+(\mu v / c) h_{\mathrm{g}}\right]
$$

$$
c^{-1}\left(\partial h_{\mathrm{g}} / \partial t\right)+\left(\partial j_{\mathrm{g}} / \partial s\right)=\kappa_{\mathrm{g}}\left[-h_{\mathrm{g}}+(\mu v / c)\left(j_{\mathrm{g}}+3 \tilde{B}_{\mathrm{g}}\right)\right],
$$

or for a fixed set of angles $\left\{\mu_{m}\right\}$, with a system of the form

$$
\frac{1}{c}\left(\frac{\partial j_{m \mathrm{~g}}}{\partial t}\right)+3 \mu_{m} \frac{\partial\left(r^{2} h_{m \mathrm{~g}}\right)}{\partial\left(r^{3}\right)}+\frac{1}{r} \sum_{m^{\prime}} D_{m m^{\prime}} h_{m^{\prime} \mathrm{g}}=\kappa_{\mathrm{g}}\left[\left(B_{\mathrm{g}}-j_{m \mathrm{~g}}\right)+\left(\frac{\mu_{m} v}{c}\right) h_{m \mathrm{~g}}\right] \text {, }
$$

and

$\frac{\mu_{m 2}}{c}\left(\frac{\partial h_{m g}}{\partial t}\right)+3 \mu_{m}^{2} \frac{\partial\left(r^{2} j_{m g}\right)}{\partial\left(r^{3}\right)}+\frac{1}{r} \sum_{m^{\prime}} D_{m m \cdot}^{\prime} \cdot j_{m^{\prime} \mathrm{g}}=\kappa_{g}\left[-\mu_{m} h_{m g}+\left(\frac{\mu_{m}^{2} v}{c}\right)\left(j_{m g}+3 \tilde{B}_{g}\right)\right]$.

Either set may be differenced as described in $\$ 83$. For $I$ radial shells, $G$ groups, and $M$ angles the computational effort for the tangent-ray method scales as $c I^{2} G$, and for the angle-differenced method as $c I G M^{3}$.

An advantage of the mixed-frame formulation is that in the formal solution one can solve the full transfer equation, which is exactly consistent with both the multigroup and integrated moment equations. Furthermore, because the spatial mesh is fixed, the radiation quantities are computed at the same set of positions and angles at each time level; no interpolations are required.

Given updated Eddington factors we can calculate revised spectral distributions from the multigroup moment equations. These equations have no frequency coupling (in contrast to their Lagrangean counterparts) hence can be solved directly with a computational effort that scales as $c I G$.

The advantages of the mixed-frame scheme are that (1) the equations have a simple structure, (2) a solution of the full transfer equation is 
possible, and (3) the multigroup equations are uncoupled. These advantages must be weighed against two serious disadvantages.

(1) Frequency derivatives of the material opacity and emissivity are required. We have avoided the issue here by assuming a constant opacity within groups. For plasmas where the opacity is dominated by light ions and is relatively smooth, reasonable estimates of the necessary derivatives may be obtainable; but for complex, jagged spectra it is extremely difficult to estimate meaningful opacity derivatives. In particular the expansion procedure used in this approach is unsatisfactory for spectral lines, which can be of great importance in media with velocity gradients. A spectral line is smeared over frequency by a velocity gradient, hence the effective widths of lines increase and continuum windows between lines are filled in, producing a substantial increase in the Rosseland mean. An interesting discussion of the problem using Sobolev theory [see (M3, Chap. 14)] is given in (K1), where velocity effects are found to be major. The problems just described are less serious in the Lagrangean frame, where the opacity is always that measured by an observer at rest relative to the material. Here the complication to be faced is the proper treatment of the $\partial\left(\nu E_{\nu}\right) / \partial \nu$ term, which is fundamentally easier because the frequency variation of $E_{\nu}$ is usually smooth (perhaps even Planckian) even when the opacity spectrum is jagged.

(2) We obtain the correct lab-frame flux only by including the two $v / c$ terms on the right-hand side of $(99.11)$, which dominate $\nabla \cdot P$ in the dynamic diffusion limit. Thus the accuracy of the computed flux hangs on obtaining an accurate representation of these terms in the difference equations, which is nontrivial because of the interleaving of the radiation variables on the grid. In contrast, in the Lagrangean formulation we obtain the correct comoving-frame flux in both the streaming and diffusion limits without any essential difficulty.

The mixed-frame equations can also be written in quasi-Lagrangean form. To illustrate the approach with a minimum of complication, we consider planar geometry, using the column mass $d m=-\rho d z$ as independent variable. Using the definition $(D / D t)=(\partial / \partial t)+\mathbf{v} \cdot \boldsymbol{\nabla}$ and the equation of continuity, one finds

$$
\left(\partial I_{\nu} / \partial t\right) \equiv \rho\left[D\left(I_{\nu} / \rho\right) / D t\right]-\nabla \cdot\left(I_{\nu} \mathbf{v}\right) .
$$

Thus the mixed-frame transfer equation in Cartesian coordinates can be written in quasi-Lagrangean form

$$
\frac{1}{c} \frac{D}{D t}\left(\frac{I_{\nu}}{\rho}\right)-\frac{\partial}{\partial m}\left[\left(\mu-\frac{v}{c}\right) I_{\nu}\right]=\left(\frac{\kappa_{\nu}}{\rho}\right)\left(B_{\nu}-I_{\nu}\right)+\left(\frac{\mu v}{c \rho}\right)\left(\tilde{\kappa}_{\nu} I_{\nu}+\eta_{\nu}\right),
$$

whence we obtain the multigroup equations

$$
\frac{1}{c} \frac{D}{D t}\left(\frac{j_{\mathrm{g}}}{\rho}\right)-\frac{\partial}{\partial m}\left(\mu h_{\mathrm{g}}-\frac{v}{c} j_{\mathrm{g}}\right)=\left(\frac{\kappa_{\mathrm{g}}}{\rho}\right)\left(B_{\mathrm{g}}-j_{\mathrm{g}}\right)+\left(\frac{\mu v}{c}\right)\left(\frac{\kappa_{\mathrm{g}}}{\rho}\right) h_{\mathrm{g}}
$$


and

$$
\frac{1}{c} \frac{D}{D t}\left(\frac{h_{\mathrm{g}}}{\rho}\right)-\frac{\partial}{\partial m}\left(\mu j_{\mathrm{g}}-\frac{v}{c} h_{\mathrm{g}}\right)=-\left(\frac{\kappa_{\mathrm{g}}}{\rho}\right) h_{\mathrm{g}}+\left(\frac{\mu v}{c}\right)\left(\frac{\kappa_{\mathrm{g}}}{\rho}\right)\left(j_{\mathrm{g}}+3 \tilde{B}_{\mathrm{g}}\right)
$$

the multigroup moment equations

$$
\frac{D}{D t}\left(\frac{E_{\mathrm{g}}}{\rho}\right)-\frac{\partial}{\partial m}\left(F_{\mathrm{g}}-v E_{\mathrm{g}}\right)=\left(\frac{\kappa_{\mathrm{g}}}{\rho}\right)\left(4 \pi B_{\mathrm{g}}-c E_{\mathrm{g}}+\frac{v}{c} F_{\mathrm{g}}\right)
$$

and

$$
\frac{1}{c^{2}} \frac{D}{D t}\left(\frac{F_{\mathrm{g}}}{\rho}\right)-\frac{\partial}{\partial m}\left(f_{\mathrm{g}} E_{\mathrm{g}}-\frac{v}{c^{2}} F_{\mathrm{g}}\right)=\frac{1}{c}\left(\frac{\kappa_{\mathrm{g}}}{\rho}\right)\left[-F_{\mathrm{g}}+v\left(f_{\mathrm{g}} E_{\mathrm{g}}+\tilde{B}_{\mathrm{g}}\right)\right]
$$

and the radiation energy and momentum equations

$$
\frac{D}{D t}\left(\frac{E}{\rho}\right)-\frac{\partial}{\partial m}(F-v E)=\frac{1}{\rho}\left(4 \pi \kappa_{P} B-c \kappa_{E} E+\frac{v}{c} \kappa_{F} F\right)
$$

and

$$
\frac{1}{c^{2}} \frac{D}{D t}\left(\frac{F}{\rho}\right)-\frac{\partial}{\partial m}\left(f E-\frac{v}{c^{2}} F\right)=\frac{1}{c \rho}\left[-\kappa_{F} F+v\left(\kappa_{K} f E+\kappa_{p} B\right)\right] .
$$

According to the dimensional arguments of $\$ 93,(99.28)$ can be simplified to

$$
c^{-2}(D F / D t)-[\partial(f E) / \partial m]=(1 / c \rho)\left[-\kappa_{F} F+v\left(\kappa_{K} f E+\kappa_{P} B\right)\right]
$$

equation (99.26) can be similarly simplified.

Equations (99.28) and (99.29) are coupled to the quasi-Lagrangean momentum equation (94.14)

$$
\frac{D v}{D t}=-g+\frac{\partial}{\partial m}(p+P)=-g+\frac{\partial p}{\partial m}+\left(\frac{1}{c \rho}\right)\left[\kappa_{F} F-v\left(\kappa_{K} f E+\kappa_{P} B\right)\right]
$$

and gas energy equation (94.19)

$$
(D e / D t)+p[D(1 / \rho) / D t]=\varepsilon+\left[c \kappa_{E} F-4 \pi \kappa_{\mathrm{P}} B-2(v / c) \kappa_{F} F\right] / \rho .
$$

The solution of (99.27) and (99.29) to (99.31) proceeds essentially as outlined in $\$ 98$. The material equations are now complicated by the presence of velocity-dependent terms. The momentum equation presents no difficulty if the first form in (99.30) is used, but in the energy equation cancellation among terms on the right-hand side may be troublesome in the diffusion regime. The quasi-Lagrangean equations (99.27) and (99.29) suffer the same disadvantages as their Eulerian counterparts (99.10) and (99.11). Moreover, in differencing $\partial(v E) / \partial m$ in (99.27) the centering is bad, 
and one may lose accuracy. The equations in spherical geometry are even messier and harder to handle well. The quasi-Lagrangean transfer equations (99.23) and (99.24) contain similar awkward terms, and the formal solution becomes complicated because now the mesh moves. One either can ignore the motions, or else must interpolate quantities at the old time level; both options introduce inaccuracies.

In summary, the mixed-frame method suffers a number of disadvantages, and while neither formulation is completely free of problems, we judge the Lagrangean approach of $\S 98$ to be physically more appealing, and both simpler and more accurate in application.

THE VERA-CODE METHOD

In the VERA-code method (F4), (P2), (S2), spacetime, angles, and frequencies are measured in the lab frame while the specific intensity and material properties are computed in the comoving frame. We will derive the equations in spherical symmetry, and then restate important results in tensor notation so that they apply in general geometries.

We start from the mixed-frame equation

$$
\begin{aligned}
\frac{1}{c} \frac{\partial I_{\mu \nu}}{\partial t}+\frac{\mu}{r^{2}} \frac{\partial}{\partial r}\left(r^{2} I_{\mu \nu}\right)+\frac{1}{r} \frac{\partial}{\partial \mu}\left[\left(1-\mu^{2}\right) I_{\mu \nu}\right] & \\
= & \eta_{\nu}^{0}-\kappa_{\nu}^{0} I_{\mu \nu}+\frac{\mu v}{c}\left[3 \eta_{\nu}^{0}-\frac{\partial}{\partial \nu}\left(\nu \eta_{\nu}^{0}\right)+I_{\mu \nu} \frac{\partial}{\partial \nu}\left(\nu \kappa_{\nu}^{0}\right)\right],
\end{aligned}
$$

and transform $I_{\mu, \nu} \equiv I(r, t ; \mu, \nu)$ to the comoving frame by a first-order expansion. Thus from (89.10), (89.11), and (90.3) we have

$$
\begin{aligned}
I_{\mu \nu}= & \left(\nu / \nu_{0}\right)^{3} I^{0}\left(\mu_{0}, \nu_{0}\right)=\left(1+\beta \mu_{0}\right)^{3}\left[I^{0}(\mu, \nu)+\left(\nu_{0}-\nu\right)\left(\partial I^{0} / \partial \nu\right)\right. \\
& \left.+\left(\mu_{0}-\mu\right)\left(\partial I^{0} / \partial \mu\right)\right]+\mathrm{O}\left(v^{2} / c^{2}\right) \\
= & (1+3 \beta \mu) I_{\mu \nu}^{0}-\beta \mu \nu\left(\partial I^{0} / \partial \nu\right)-\beta\left(1-\mu^{2}\right)\left(\partial I^{0} / \partial \mu\right)+\mathrm{O}\left(v^{2} / c^{2}\right) .
\end{aligned}
$$

More generally,

$$
I(\mathbf{n}, \nu)=[1+(3 \mathbf{v} \cdot \mathbf{n} / c)] I^{\mathrm{O}}(\mathbf{n}, \nu)-(\mathbf{v} \cdot \mathbf{n} / c) \nu\left(\partial I^{\mathrm{0}} / \partial \nu\right)-(\mathbf{v} / c) \cdot \nabla_{\mathbf{n}} I^{\mathrm{0}}
$$

where $\nabla_{\mathbf{n}}$ denotes the gradient with respect to the direction cosines of the propagation vector.

Integrating (99.33) over solid angle we obtain transformation laws for the monochromatic radiation moments:

$$
\begin{aligned}
& E_{\nu}=E_{\nu}^{0}+\left(2 v / c^{2}\right) F_{\nu}^{0}-\left(v / c^{2}\right)\left[\partial\left(\nu F_{\nu}^{0}\right) / \partial \nu\right], \\
& F_{\nu}=F_{\nu}^{0}+v\left(E_{\nu}^{0}+P_{\nu}^{0}\right)-v\left[\partial\left(\nu P_{\nu}^{0}\right) / \partial \nu\right],
\end{aligned}
$$

and

$$
P_{\nu}=P_{\nu}^{0}+\left(2 v / c^{2}\right) F_{\nu}^{0}-\left(v / c^{2}\right)\left[\partial\left(\nu Q_{\nu}^{0}\right) / \partial \nu\right]
$$


Or, in tensor form

$$
\begin{aligned}
& E_{\nu}=E_{\nu}^{0}+\left(2 / c^{2}\right) \mathbf{v} \cdot \mathbf{F}_{\nu}^{0}-c^{-2}\left[\partial\left(\nu \mathbf{v} \cdot \mathbf{F}_{\nu}^{0}\right) / \partial \nu\right], \\
& \mathbf{F}_{\nu}=\mathbf{F}_{\nu}^{0}+\mathbf{v} E_{\nu}^{0}+\mathbf{v} \cdot \mathbf{P}_{\nu}^{0}-\left[\partial\left(\nu \mathbf{v} \cdot \mathbf{P}_{\nu}^{0}\right) / \partial \nu\right],
\end{aligned}
$$

and

$$
\mathbf{P}_{\nu}=\mathbf{P}_{\nu}^{0}+c^{-2}\left(\mathbf{v} \mathbf{F}_{\nu}^{0}+\mathbf{F}_{\nu}^{0} \mathbf{v}\right)-c^{-2}\left[\partial\left(\nu \mathbf{v} \cdot \mathbf{Q}_{\nu}^{0}\right) / \partial \nu\right]
$$

where

$$
\mathrm{Q}_{\nu}^{\circ} \equiv \oint I^{\circ}(\mathbf{n}, \nu) \mathbf{n n n} d \omega
$$

One sees by inspection that (99.35) to $(99.40)$, when integrated over frequency yield the standard $\mathrm{O}(v / c)$ transformations of the radiation stressenergy tensor [cf. (91.10) to (91.12) and (91.16) to (91.18)].

Substituting (99.33) into (99.32), and using (99.21), we obtain the transfer equation

$$
\begin{aligned}
\frac{\rho}{c} & \frac{D}{D t}\left(\frac{I_{\mu \nu}^{0}}{\rho}\right)+\frac{\mu}{r^{2}} \frac{\partial}{\partial r}\left(r^{2} I_{\mu \nu}^{0}\right)+\frac{1}{r} \frac{\partial}{\partial \mu}\left[\left(1-\mu^{2}\right) I_{\mu \nu}^{0}\right]=\eta_{\nu}^{0}-\kappa_{\nu}^{0} I_{\mu \nu}^{0}+\frac{1}{c r^{2}} \frac{\partial}{\partial r}\left(r^{2} v I_{\mu \nu}^{0}\right) \\
& -\frac{1}{c} \llbracket\left[\kappa_{\nu}^{0}+\frac{1}{c} \frac{\partial}{\partial t}+\frac{\mu}{r^{2}} \frac{\partial}{\partial r} r^{2}+\frac{1}{r} \frac{\partial}{\partial \mu}\left(1-\mu^{2}\right)\right]\left\{\mu v\left[2 I_{\mu \nu}^{0}-\frac{\partial}{\partial \nu}\left(\nu I_{\mu \nu}^{0}\right)\right]\right. \\
& \left.\left.-v \frac{\partial}{\partial \mu}\left[\left(1-\mu^{2}\right) I_{\mu \nu}^{0}\right]\right\}-\mu v\left[3 \eta_{\nu}^{0}-\frac{\partial}{\partial \nu}\left(\nu \eta_{\nu}^{0}\right)+I_{\mu \nu}^{0} \frac{\partial}{\partial \nu}\left(\nu \kappa_{\nu}^{0}\right)\right]\right]+\mathrm{O}\left(v^{2} / c^{2}\right)
\end{aligned}
$$

Taking the zeroth angular moment of (99.42), we obtain, after some reduction,

$$
\begin{aligned}
\rho \frac{D}{D t} & \left(\frac{E_{\nu}^{0}}{\rho}\right)+\frac{1}{r^{2}} \frac{\partial}{\partial r}\left[r^{2}\left(F_{\nu}^{0}+v P_{\nu}^{0}\right)\right]-4 \pi \eta_{\nu}^{0}+c \kappa_{\nu}^{0} E_{\nu}^{0}+\frac{v}{c} \kappa_{\nu}^{0} F_{\nu}^{0} \\
& =\frac{\partial}{\partial \nu}\left\{\nu\left[\frac{v}{c} \kappa_{\nu}^{0} F_{\nu}^{0}+\frac{1}{c^{2}} \frac{\partial}{\partial t}\left(v F_{\nu}^{0}\right)+\frac{1}{r^{2}} \frac{\partial}{\partial r}\left(r^{2} v P_{\nu}^{0}\right)\right]\right\}-\frac{2}{c^{2}} \frac{\partial}{\partial t}\left(v F_{\nu}^{0}\right)
\end{aligned}
$$

or, more generally,

$$
\begin{aligned}
& \rho\left[D\left(E_{\nu}^{0} / \rho\right) / D t\right]+\boldsymbol{\nabla} \cdot\left(\mathbf{F}_{\nu}^{0}+\mathbf{v} \cdot \mathbf{P}_{\nu}^{0}\right)-4 \pi \eta_{\nu}^{0}+c \kappa_{\nu}^{0} E_{\nu}^{0}+\left(\kappa_{\nu}^{0} / c\right) \mathbf{v} \cdot \mathbf{F}_{\nu}^{0} \\
& \quad=\frac{\partial}{\partial \nu}\left\{\nu\left[\frac{\kappa_{v}^{0}}{c} \mathbf{v} \cdot \mathbf{F}_{\nu}^{0}+\frac{1}{c^{2}} \frac{\partial}{\partial t}\left(\mathbf{v} \cdot \mathbf{F}_{\nu}^{0}\right)+\nabla \cdot\left(\mathbf{v} \cdot \mathbf{P}_{\nu}^{0}\right)\right]\right\}-\frac{2}{c^{2}} \frac{\partial}{\partial t}\left(\mathbf{v} \cdot \mathbf{F}_{v}^{0}\right) .
\end{aligned}
$$

Similarly, after rearrangement the first moment of (99.42) can be written

$$
\begin{aligned}
& \frac{\rho}{c^{2}} \frac{D}{D t}\left(\frac{F_{v}^{0}}{\rho}\right)+\frac{\partial P_{\nu}^{0}}{\partial r}+\frac{3 P_{\nu}^{0}-E_{\nu}^{0}}{r}+\frac{\kappa_{\nu}^{0} F_{\nu}^{0}}{c}=-\frac{1}{c^{2}}\left[E_{\nu}^{0} \frac{\partial v}{\partial t}+\frac{\partial}{\partial t}\left(v P_{\nu}^{0}\right)+F_{\nu}^{0} \frac{\partial v}{\partial r}\right] \\
& +\frac{1}{c^{2}} \frac{\partial}{\partial \nu}\left\{\nu\left[\frac{\partial}{\partial t}\left(v P_{\nu}^{0}\right)+\frac{\partial}{\partial r}\left(v Q_{\nu}^{0}\right)+\frac{v\left(3 Q_{\nu}^{0}-F_{\nu}^{0}\right)}{r}+v\left(c \kappa_{\nu}^{0} P_{\nu}^{0}-\frac{4}{3} \pi \eta_{v}^{0}\right)\right]\right\},
\end{aligned}
$$


or, in tensor form,

$$
\begin{aligned}
\frac{\rho}{c^{2}} \frac{D}{D t}\left(\frac{\mathbf{F}_{\nu}^{0}}{\rho}\right)+\boldsymbol{\nabla} \cdot \mathrm{P}_{\nu}^{0}+\frac{\kappa_{v}^{0}}{c} \mathbf{F}_{\nu}^{0}=-\frac{1}{c^{2}}\left[E_{\nu}^{0} \frac{\partial \mathbf{v}}{\partial t}+\frac{\partial\left(\mathbf{v} \cdot \mathbf{P}_{\nu}^{0}\right)}{\partial t}+\mathbf{F}_{\nu}^{0} \cdot \boldsymbol{\nabla} \mathbf{v}\right] \\
+\frac{1}{c^{2}} \frac{\partial}{\partial \nu}\left\{\nu\left[\frac{\partial}{\partial t}\left(\mathbf{v} \cdot \mathbf{P}_{\nu}^{0}\right)+\boldsymbol{\nabla} \cdot\left(\mathbf{v} \cdot \mathrm{Q}_{\nu}^{0}\right)+c \kappa_{\nu}^{0} \mathbf{v} \cdot \mathbf{P}_{\nu}^{0}-\frac{4 \pi}{3} \eta_{\nu}^{0} \mathbf{v}\right]\right\} .
\end{aligned}
$$

In deriving (99.45) and (99.46) the first term on the right was reduced by use of (99.43) and (99.44).

Integrating (99.44) and (99.46) over frequency we obtain the radiation energy equation

$$
\begin{array}{r}
\rho\left[D\left(E^{0} / \rho\right) / D t\right]+\boldsymbol{\nabla} \cdot\left(\mathbf{F}^{0}+\mathbf{v} \cdot \mathrm{P}^{0}\right)-4 \pi \kappa_{P}^{0} B^{0}+c \kappa_{E}^{0} E^{0}+\left(\kappa_{F}^{0} / c\right) \mathbf{v} \cdot \mathbf{F}^{0} \\
=-\left(2 / c^{2}\right) \partial\left(\mathbf{v} \cdot \mathbf{F}^{0}\right) / \partial t
\end{array}
$$

and the radiation momentum equation

$$
\begin{aligned}
\left(\rho / c^{2}\right)\left[D\left(\mathbf{F}^{0} / \rho\right) / D t\right]+ & \nabla \cdot P^{0}+\left(\kappa_{F}^{0} / c\right) \mathbf{F}^{0} \\
& =-c^{-2}\left[E^{0}(\partial \mathbf{v} / \partial t)+\partial\left(\mathbf{v} \cdot P^{0}\right) / \partial t+\mathbf{F}^{0} \cdot \nabla \mathbf{v}\right] .
\end{aligned}
$$

Despite its rather different appearance, (99.47) can be reduced to the Lagrangean radiation energy equation (95.87). To see this we expand

$$
\partial\left(\mathbf{v} \cdot \mathbf{F}^{o}\right) / \partial t=\mathbf{a} \cdot \mathbf{F}+\mathbf{v} \cdot\left(\partial \mathbf{F}^{\mathrm{o}} / \partial t\right)
$$

and

$$
\boldsymbol{\nabla} \cdot\left(\mathbf{v} \cdot \mathrm{P}^{0}\right)=\mathrm{P}^{0}: \boldsymbol{\nabla} \mathbf{v}+\mathbf{v} \cdot\left(\boldsymbol{\nabla} \cdot \mathrm{P}^{0}\right)
$$

whence we find, after rearrangement,

$$
\begin{aligned}
\rho \frac{D}{D t}\left(\frac{E_{0}}{\rho}\right)+ & \boldsymbol{\nabla} \cdot \mathbf{F}^{0}+\frac{\mathbf{v}}{c^{2}} \cdot \frac{\partial \mathbf{F}^{0}}{\partial t}+\mathrm{P}^{0}: \nabla \mathbf{v}+\frac{2}{c^{2}} \mathbf{a} \cdot \mathbf{F}^{0} \\
& =4 \pi \kappa_{P}^{0} B^{0}-c \kappa_{E}^{0} E^{0}-\mathbf{v} \cdot\left(\frac{1}{c^{2}} \frac{\partial \mathbf{F}^{0}}{\partial t}-\nabla \cdot P^{0}+\frac{\kappa_{F}^{0}}{c} \mathbf{F}^{0}\right) .
\end{aligned}
$$

The last term on the right-hand side of $(99.51)$ vanishes to $\mathrm{O}\left(v^{2} / c^{2}\right)$ by virtue of (99.48). Thus (99.51) differs from (95.87) only by the term $c^{-2} \mathbf{v} \cdot\left(\partial \mathbf{F}^{\circ} / \partial t\right)$ on the left-hand side. This term is present because the operator $\boldsymbol{\nabla}$ in (99.51) is in the Eulerian frame, whereas in (95.87) it is in the Lagrangean frame. From (92.12) [see also (B2, equation 2)] one has, to $\mathrm{O}(v / c)$

$$
\boldsymbol{\nabla}_{E}=\boldsymbol{\nabla}_{L}-c^{-2} \mathbf{v}(\partial / \partial t),
$$

whence we see that (99.51) and (95.87) are physically equivalent. Nevertheless, one should note that although $(99.47)$ reduces algebraically 
to (95.87), from a computational point of view it is quite different, being more difficult to handle well, and probably yielding less accurate results.

By a similar rearrangement of $(99.48)$ we find

$$
\frac{\rho}{c^{2}} \frac{D}{D t}\left(\frac{\mathbf{F}^{0}}{\rho}\right)+\nabla \cdot \mathrm{P}^{0}+\frac{\mathbf{v}}{c^{2}} \cdot \frac{\partial \mathrm{P}^{0}}{\partial t}+\mathbf{F}^{\circ} \cdot \nabla \mathbf{v}+\frac{1}{c^{2}}\left(E^{0} \frac{\partial \mathbf{v}}{\partial t}+\mathbf{P}^{0} \cdot \frac{\partial \mathbf{v}}{\partial t}\right)+\frac{\kappa_{F} \mathbf{F}^{\circ}}{c}=0
$$

which, by virtue of $(99.52)$, reduces to $(95.88)$.

Equations (99.47) and (99.48) are closed with variable Eddington factors and then coupled to Lagrangean material momentum and energy equations such as (98.14) and (98.15); the combined system is solved by the multifrequency/grey technique as described in $\$ 98$. (Indeed, the VERA code is the original source of this method.) Difference representations of the VERA equations are thoroughly documented in (F4), (P2), and (S2); here we merely critique the computational procedure.

The VERA equations are more complicated than their Lagrangean counterparts, and many approximations were made in implementing them. Thus in solving (99.47) and (99.48) the VERA code drops all the terms on the right-hand sides of these equations. Analysis shows that these terms are at most $\mathrm{O}(\mathrm{v} / \mathrm{c})$ relative to the dominant terms, hence their omission is justified. Equation (99.48) is then essentially identical to our Lagrangean equation (98.8). In (99.47) the term $\mathbf{v} \cdot \mathrm{P}^{0}$ has an awkward centering relative to $\mathbf{F}^{0}$, as does $\mathbf{v} \cdot \mathbf{F}^{0}$ relative to $E^{0}$. In retrospect one realizes that these terms should have been expanded and canceled as in (99.51), which would have yielded a simpler, fully Lagrangean equation.

The situation for the multigroup equations is worse; all terms on the right-hand sides of (99.44) and (99.46) were dropped. These omissions are harmless in (99.46). Most of the terms omitted from (99.44) can be dropped without ill effect, but it is important to retain $\partial\left(\nu \boldsymbol{\nabla} \cdot \mathbf{v} \cdot \mathrm{P}_{\nu}^{0}\right) / \partial \nu$ in order to obtain the correct spectral distribution (cf. \$97). Again the Lagrangean equation (98.1) is both simpler and more accurate.

Finally, in the formal solution, it is clear that a rigorous treatment of (99.42) is hopeless. Early versions of the VERA code (F4) used formulae of the kind mentioned in $\$ 78$ to evaluate the Eddington factor from $E^{0}$ and $F^{0}$. Later versions $[(\mathbf{F 5}),(\mathbf{P 2}),(\mathbf{S 2})]$ evaluated $f$ from either time-retarded solutions or static snapshots along tangent rays, omitting all velocitydependent terms. As was argued in $\$ 98$, this approach should be adequate in many applications, but it is less reliable and satisfying than the Lagrangean methods sketched in $(98.50)$ to $(98.58)$, or the mixed-frame approach outlined in (99.17) to $(99.20)$.

In summary, the VERA equations resemble the Lagrangean equations, but are more complicated and more difficult to solve to a high level of internal consistency; the Lagrangean equations are to be preferred [see also (B2, pp. 298-299)]. 


\section{References}

(A1) Alme, M. L. and Wilson, J. R. (1974) Astrophys. J., 194, 147.

(B1) Barfield, W. D., von Holdt, R., and Zachariasen, F. (1954) Los Alamos Scientific Laboratory Report No. LA-1709. Los Alamos: University of California.

(B2) Buchler, J. R. (1979) J. Quant. Spectrosc. Rad. Transf., 22, 293.

(B3) Buchler, J. R. (1983) J. Quant. Spectrosc. Rad. Transf., 30, 395.

(C1) Campbell, P. M. (1965) Lawrence Radiation Laboratory Report No. UCRL12411. Livermore: University of California.

(C2) Campbell, P. M. and Nelson, R. G. (1964) Lawrence Radiation Laboratory Report No. UCRL-7838. Livermore: University of California.

(C3) Castor, J. I. (1972) Astrophys. J., 178, 779.

(C4) Christy, R. F. (1964) Rev. Mod. Phys., 36, 555.

(C5) Cox, J. P. and Giuli, R. T. (1968) Principles of Stellar Structure. New York: Gordon and Breach.

(E1) Epstein, R. 1. (1981) Astrophys. J. Letters, 244, L89.

(F1) Falk, S. W. and Arnett, W. D. (1977) Astrophys. J. Supp., 33, 515.

(F2) Fraser, A. R. (1966) Atomic Weapons Research Establishment Report No. O-82/65. Aldermaston: U.K. Atomic Energy Authority.

(F3) Freeman, B. E. (1965) Los Alamos Scientific Laboratory Report No. LA3377, Los Alamos: University of California.

(F4) Freeman, B. E., Hauser, L. E., Palmer, J. T., Pickard, S. O., Simmons, G. M., Williston, D. G., and Zerkle, J. E. (1968) Defense Atomic Support Agency Report No. DASA 2135, Vol. 1. La Jolla: Systems, Science, and Software, Inc.

(F5) Freeman, B. E., Palmer, J. T., Simmons, G. M., Schaibly, J. H., and Zerkle, J. E. (1969) Defense Atomic Support Agency Report No. DASA 2258-III. La Jolla: Systems, Science, and Software, Inc.

(G1) Glaviano, M. C. and Raymond, D. J. (1981) Astrophys. J., 243, 271.

(H1) Hazelhurst, J. and Sargent, W. L. W. (1959) Astrophys. J., 130, 276.

(H2) Hsieh, S.-H. and Spiegel, E. A. (1976) Astrophys. J., 207, 244.

(J1) Jeans, J. H. (1926) Mon. Not. Roy. Astron. Soc., 86, 328.

(J2) Jeans, J. H. (1926) Mon. Not. Roy. Astron. Soc., 86, 444.

(K1) Karp, A. H., Lasher, G., Chan, K. L., and Salpeter, E. E. (1977) Astrophys. J., 214, 161.

(K2) Kershaw, D. S. (1976) Lawrence Livermore Laboratory Report No. UCRL78378. Livermore: University of California.

(K3) Klein, R. I., Stockman, H. S., and Chevalier, R. A. (1980) Astrophys. J., 237, 921.

(K4) Kneer, F. and Nakagawa, Y. (1976) Astron. and Astrophys., 47, 65.

(K5) Kopal, Z. (1964) Astrophysica Norvegica, 9, 239.

(K6) Kopal, Z. (1965) Z. für Astrophys., 61, 156.

(K7) Kutter, G. S. and Sparks, W. M. (1972) Astrophys. J., 175, 407.

(L1) Ledoux, P. (1965) in Stellar Structure, ed. L. H. Aller and D. B. McLaughlin, Chapter 10. Chicago: University of Chicago.

(L2) Ledoux, P. and Walraven, Th. (1958) in Handbuch der Physik, Vol. 51, Astrophysics II: Stellar Structure, ed. S. Flügge, p. 353. Berlin: Springer.

(L3) Levermore, C. D. (1979) Lawrence Livermore Laboratory Report No. UCID-18229. Livermore: University of California. 
(L4) Levermore, C. D. and Pomraning, G. C. (1981) Astrophys. J. 248, 321.

(L5) Lindquist, R. W. (1966) Ann. Phys., 37, 487.

(M1) Masaki, I. (1971) Pub. Astron. Soc. Japan, 23, 425.

(M2) Masaki, I. (1981) Pub. Astron. Soc. Japan, 33, 77.

(M3) Mihalas, D. (1978) Stellar Atmospheres. (2nd ed.) San Francisco: Freeman.

(M4) Mihalas, D. (1980) Astrophys. J., 237, 574.

(M5) Mihalas, D. (1980) Astrophys. J., 238, 1042.

(M6) Mihalas, D. (1981) Astrophys. J., 250, 373.

(M7) Mihalas, D. (1983) Astrophys. J., 266, 242.

(M8) Mihalas, D. and Klein, R. I. (1982) J. Comp. Phys, 46, 97.

(M9) Mihalas, D. and Kunasz, P. B. (1978) Astrophys. J., 219, 635.

(M10) Mihalas, D. and Weaver, R. P. (1982) J. Quant. Spectrosc. Rad. Transf., 28, 213.

(M11) Mihalas, D., Kunasz, P. B., and Hummer, D. G. (1975) Astrophys. J., 202, 465.

(M12) Mihalas, D., Kunasz, P. B., and Hummer, D. G. (1976) Astrophys. J., 206, 515.

(M13) Mihalas, D., Kunasz, P. B., and Hummer, D. G. (1976) Astrophys. J., 210, 419.

(M14) Milne, E. A. (1929) Mon. Not. Roy. Astron. Soc., 89, 519.

(M15) Milne, E. A. (1930) Quart. J. of Math. (Oxford), 1, 1.

(M16) Morse, P. M. and Feshbach, H. (1953) Methods of Theoretical Physics. New York: McGraw-Hill.

(N1) Noerdlinger, P. D. (1981) Astrophys. J., 245, 682.

(P1) Pai, S. I. (1966) Radiation Gas Dynamics. New York: Springer.

(P2) Palmer, J. T., Freeman, B. E., and Schaibly, J. H. (1969) Defense Atomic Support Agency Report No. DASA 2420-1. La Jolla: Systems, Science, and Software, Inc.

(P3) Pomraning, G. C. (1973) The Equations of Radiation Hydrodynamics. Oxford: Pergamon.

(P4) Pomraning, G. C. (1974) I. Quant. Spectrosc. Rad. Transf., 14, 657.

(R1) Richtmyer, R. D. and Morton, K. W. (1967) Difference Methods for InitialValue Problems. (2nd ed.) New York: Interscience.

(R2) Roache, P. J. (1976) Computational Fluid Dynamics. Albuquerque: Hermosa.

(S1) Sampson, D. H. (1965) Radiative Contributions to Energy and Momentum Transport in a Gas. New York: Interscience.

(S2) Schaibly, J. and Wilson, A. R. (1972) Systems, Science, and Software Report No. 3SCR-1449. La Jolla: Systems, Science, and Software, Inc.

(S3) Sears, R. L. and Brownlee, R. R. (1965) in Stellar Structure, ed. L. H. Aller and D. B. McLaughlin, Chapter 11. Chicago: University of Chicago.

(S4) Simon, R. (1963) J. Quant. Spectrosc. Rad. Transf., 3, 1.

(S5) Stobie, R. S. (1969) Mon. Not. Roy. Astron. Soc., 144, 461.

(S6) Synge, J. L. (1957) The Relativistic Gas. Amsterdam: North Holland.

(S7) Synge, J. L. and Schild, A. R. (1949) Tensor Calculus. Toronto: University of Toronto.

(T1) Thomas, L. H. (1930) Quart. J. of Math. (Oxford), 1, 239.

(T2) Tisza, L. (1942) Phys. Rev. 61, 531.

(T3) Tscharnuter, W. and Winkler, K.-H. (1969) Computer Phys. Comm., 18, 171. 
(W1) Weinberg, S. (1971) Astrophys. J., 168, 175.

(W2) Wendroff, B. (1963) Los Alamos Scientific Laboratory Report No. LAMS2795. Los Alamos: University of California.

(W3) Winkler, K.-H. and Norman, M. (1984) in Astrophysical Radiation Hydrodynamics, ed. K.-H. Winkler and M. Norman. Dordrecht: Reidel.

(W4) Winslow, A. M. (1968) Nucl. Sci. and Eng., 32, 101.

(Z1) Zel'dovich, Ya. B. and Raizer, Yu. P. (1966) Physics of Shock Waves and High-Temperature Hydrodynamic Phenomena. New York: Academic. 